\title{
Fading Broadcast Channels with State Information at the Receivers
}

\author{
David Tse* Roy Yates ${ }^{\dagger}$
}

April 20, 2009

\begin{abstract}
Despite considerable progress on the information-theoretic broadcast channel, the capacity region of fading broadcast channels with channel state known at the receivers but unknown at the transmitter remains unresolved. We address this subject by introducing a layered erasure broadcast channel model in which each component channel has a state that specifies the received signal levels in an instance of a deterministic binary expansion channel. We find the capacity region of this class of broadcast channels. The capacity achieving strategy assigns each signal level to the user that derives the maximum expected rate from that level. The outer bound is based on a channel enhancement that creates a degraded broadcast channel for which the capacity region is known. This same approach is then used to find inner and outer bounds to the capacity region of fading Gaussian broadcast channels. The achievability scheme employs a superposition of binary inputs. For intermittent AWGN channels and for Rayleigh fading channels, the achievable rates are observed to be with 1-2 bits of the outer bound at high SNR. We also prove that the achievable rate region is within $6.386 \mathrm{bits} / \mathrm{s} / \mathrm{Hz}$ of the capacity region for all fading AWGN broadcast channels.
\end{abstract}

\section{Introduction}

The two most basic multiuser communication scenarios are many-to-one and one-tomany, captured by the multiple access channel (MAC) and the broadcast channel (BC) respectively. While the capacity region of the general multiple access channel is known since the 70's $[1,2]$, that of the general broadcast channel is still open. Yet,

*D. Tse (email: dtse@eecs.berkeley.edu) is with Wireless Foundations, EECS Dept., University of California at Berkeley and acknowledges the support of NSF grant CNS-0722032.

${ }^{\dagger} \mathrm{R}$. Yates (email: ryates@winlab.rutgers.edu) is with WINLAB, E\&CE Dept., Rutgers University and acknowledges the support of NSF grant CNS-0721826. 
progress has been made in the past 40 years on special cases. A class of channels, of particular importance to wireless communication, are the Gaussian broadcast channels (AWGN BC). The capacity region of AWGN BC's is known, for both the case when the channel state is fixed and time-invariant, and for the case when the channelstate is time-varying (fading). This is true for single antenna [3-7] or for multiple antenna channels [8]. The key assumption behind these results is that the channel state is known at the transmitter as well as the receivers (perfect CSI). However, the problem becomes open once the assumption of CSI at the transmitter is removed, even when the transmitter and each of the receivers has only a single antenna. This is an important scenario in practice since in a fast fading environment, it may be difficult to feedback channel state information in a timely fashion to the transmitter from the receivers. Moreover, most cellular systems operate on a frequency-division duplex (FDD) mode rather than on a time-division duplex (TDD) mode, so the downlink channel information cannot be inferred from uplink channel measurements. Nevetheless, despite its apparent practical importance, there are very few results on the capacity of the fading $\mathrm{BC}$ with receiver-only CSI (see for example $[9,10]$ ).

The channels mentioned above for which the capacity region is known are either degraded (in the case of the single-antenna time-invariant channel), parallel with degraded components (time-varying single-antenna channels with perfect CSI) or have a related degraded structure (MIMO BC's). The fading broadcast channel with only receiver CSI has no such degraded structure for arbitrary fading distributions, thus making it a challenging problem from a theoretical standpoint.

In this paper, we focus on the simplest scenario with two receivers and a single antenna at the transmitter and at each of the receivers. Our main contribution is two-fold:

- We propose a layered erasure broadcast channel to approximate the Gaussian fading channel and determine its capacity region exactly. The erasures in this model are correlated, and, like the Gaussian fading BC, the layered erasure BC is neither degraded nor parallel with degraded components.

- Using the insights from the erasure model, we derive a new outer bound to the Gaussian fading BC capacity region and demonstrate a binary expansion superposition (BES) scheme that achieves rates within 6.386 bits $/ \mathrm{s} / \mathrm{Hz}$ per user to the outer bound. This gap holds in the worst case over all fading distributions. We also demonstrate example fading distributions for which the gap is much smaller.

The layered erasure BC is based on a new point-to-point erasure channel model. This model provides a simpler way of thinking about fading and may be of independent interest. The transmitted signal is thought of as a vector of bits, from the most significant to less significant bits. The bits can be viewed as layers of the transmitted signal. Fading is modeled as erasures of the less significant bits, and how many bits are erased depends on the instantaneous channel strength. Erasures are correlated 
because when a bit is erased, all the less significant bits are also erased. The layered erasure model can be thought of as a time-varying version of the binary expansion deterministic channel model introduced by Avestimehr, Diggavi and Tse [11]. While modeling fading as erasures has appeared in the literature (see for example [12]), typically these models regard the entire transmitted signal as erased and thus cannot capture the continuous nature of the channel strength in the Gaussian model. We do note, however, that this fading model has appeared in the control literature [13].

\section{Background and Definitions}

As introduced by Cover [3], the two-user memoryless broadcast channel (BC) consisting of a transmitter with input $X$ and receiver observations $Y_{1}$ and $Y_{2}$ described by a channel transition probability $P_{Y_{1}, Y_{2} \mid X}\left(y_{1}, y_{2} \mid x\right)$. Through this multiuser channel, the sender wishes to communicate private messages at rate $R_{i}$ to receiver $i$ as well as a common message at rate $R_{0}$ to both receivers.

In this work, we focus on the $R_{0}=0$ case where there is no common message. Even in this case, the general BC capacity region is unknown. However, the capacity region of the important special case of the degraded channel is known. A broadcast channel $P_{Y_{1}, Y_{2} \mid X}$ is degraded if there exists a Markov chain $X-Y_{1}-Y_{2}$ that yields the marginal conditional distributions $P_{Y_{1} \mid X}\left(y_{1} \mid x\right)$ and $P_{Y_{2} \mid X}\left(y_{2} \mid x\right)$ consistent with $P_{Y_{1}, Y_{2} \mid X}$. The capacity region $\mathcal{R}$ of the degraded memoryless $\mathrm{BC}[4,14]$ is given in the following theorem which we restate here.

Theorem 1 The capacity region $\mathcal{R}$ of the degraded memoryless $B C P_{Y_{1}, Y_{2} \mid X}$ is the union over all $V, X$ such that $V-X-Y_{1} Y_{2}$ of rate pairs $\left(R_{1}, R_{2}\right)$ satisfying

$$
\begin{aligned}
& R_{1} \leq I\left(X ; Y_{1} \mid V\right), \\
& R_{2} \leq I\left(V ; Y_{2}\right)
\end{aligned}
$$

To characterize the boundary of the capacity region $\mathcal{C}$ of a particular $\mathrm{BC}$, we define the weighted sum rate maximization problem

$$
\max _{\left(R_{1}, R_{2}\right) \in \mathcal{C}} \omega_{1} R_{1}+\omega_{2} R_{2} .
$$

To find the rate region $\mathcal{C}$, we define $\omega=\omega_{2} / \omega_{1}$ and we solve

$$
R^{*}(\omega)=\max _{\left(R_{1}, R_{2}\right) \in \mathcal{C}} R_{1}+\omega R_{2}
$$

For each $\omega$, the solution will be associated with a pair $\left(R_{1}^{*}, R_{2}^{*}\right)$ that defines a capacity region constraint

$$
R_{1}+\omega R_{2} \leq R_{1}^{*}+\omega R_{2}^{*}, \quad\left(R_{1}, R_{2}\right) \in \mathcal{C} .
$$

At $\omega=0$, we obtain $\left(R_{1}^{*}, R_{2}^{*}\right)=\left(C_{1}, 0\right)$ and the constraint $R_{1} \leq C_{1}$ where $C_{1}$ is the ergodic capacity of the fading channel to receiver 1 . Similarly, as $\omega \rightarrow \infty$, we obtain 


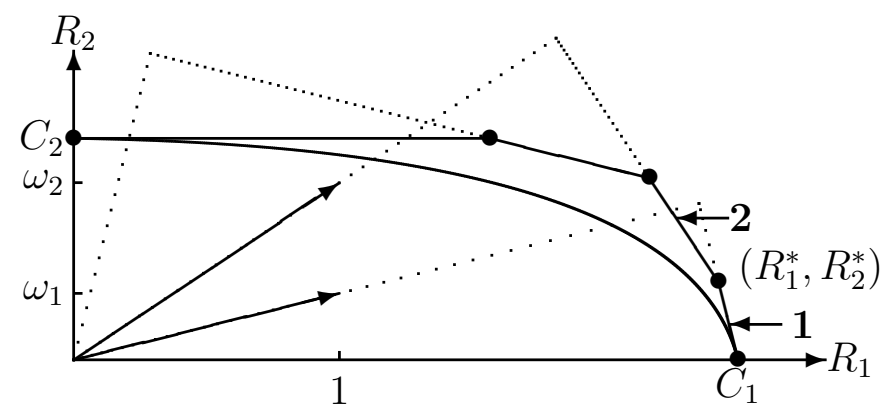

Figure 1: In the outer bound to a capacity region, each extreme point, denoted by -, of an outer bound region is specified by a pair of constraints. In this example, the boundary segments marked 1 and $\mathbf{2}$ correspond to the constraint (4) with $\omega=\omega_{1}$ and $\omega=\omega_{2}$ respectively.

the corner point $\left(R_{1}^{*}, R_{2}^{*}\right)=\left(0, C_{2}\right)$ corresponding to the ergodic capacity constraint on $R_{2}$. In general, however, we will obtain an outer bound such that the constraint (4) may not be tight.

When the channel state distributions are discrete, the point $\left(R_{1}^{*}, R_{2}^{*}\right)$ generally will be associated with an interval $\left[\omega_{1}, \omega_{2}\right]$ of weights and a family of constraints

$$
R_{1}+\omega R_{2} \leq R_{1}^{*}+\omega R_{2}^{*}, \quad\left(R_{1}, R_{2}\right) \in \mathcal{C}, \omega \in\left[\omega_{1}, \omega_{2}\right] .
$$

In this case, as shown in Figure 1, the endpoint weights $\omega_{1}$ and $\omega_{2}$ define a pair of constraints such that $\left(R_{1}^{*}, R_{2}^{*}\right)$ is an extreme point of an outer bound region. The outer bound region will be the convex hull of the set of extreme points and the origin $(0,0)$.

In our subsequent derivations, we assume $\omega \geq 1$; i.e., bits communicated to receiver 2 are favored. For the case $\omega<1$, we factor $\omega$ out of (3) and we see that the boundary of the capacity region for $\omega<1$ is given by the optimization problem

$$
R^{*}(\omega)=\omega \max _{\left(R_{1}, R_{2}\right) \in \mathcal{R}}(1 / \omega) R_{1}+R_{2}
$$

In this case, we have the identical optimization as in (3) but with the roles of users 1 and 2 reversed in that we now favor receiver 1 by the weight $1 / \omega$. However, as the labeling of receivers 1 and 2 is arbitrary, we can simply reverse label the receivers and solve (3) with $1 / \omega \geq 1$. Henceforth, our derivations using the weighted sum rate maximization (3) will assume a weight $\omega \geq 1$. We will see that the solution will remain valid for $\omega<1$.

In this paper, we adopt several conventions. For a random variable $N$, the probability mass function (PMF) is $P_{N}(n):=P[N=n]$ and the complementary cumulative distribution function $(\mathrm{CDF})$ is $\bar{F}_{N}(n):=P[N \geq n]$. The $\mathcal{N}(0,1)$ random variable $Z$ has complementary $\operatorname{CDF} Q(z)=P[Z \geq z]$. We define $\lfloor x\rfloor$ as the largest integer less than or equal $x,\lceil x\rceil$ as the smallest integer greater than or equal to $x$, and 
$x^{+}:=\max (0, x)$. We also define $\operatorname{sgn}(x)$ as the sign of $x$. That is, $\operatorname{sgn}(x)=-1$ for $x<0$ and $\operatorname{sgn}(x)=1$ for $x \geq 0$. All logarithms are to the base 2 unless otherwise noted. Proofs appear in the appendix.

\section{Layered Erasure Broadcast Channel}

\subsection{Channel Model}

We start by reviewing the broadcast channel formulation of the binary expansion deterministic channel model of [11]. Each communication channel from the sender to a receiver $j$ is associated with a non-negative integer gain $n_{j}$ that describes how many signal "levels" are observed at receiver $j$. The best channel in the system supports $q=\max _{j} n_{j}$ levels. At each time $t$, the sender transmits a vector $\mathbf{X}_{i}([t]) \in \mathbb{F}_{2}^{q}$. Algebraic definition of the received signals is based on the $q \times q$ "shift" matrix

$$
\mathbf{S}=\left[\begin{array}{ccccc}
0 & 0 & 0 & \cdots & 0 \\
1 & 0 & 0 & \cdots & 0 \\
0 & 1 & 0 & \cdots & 0 \\
\vdots & \ddots & \ddots & \ddots & \vdots \\
0 & \cdots & 0 & 1 & 0
\end{array}\right]
$$

For example, if $\mathbf{Y}=\mathbf{S}^{2} \mathbf{X}$, we have that $Y_{1}=Y_{2}=0$, and $Y_{k}=X_{k-2}$ for $k=3, \ldots, q$. We note that $\mathbf{S}^{0}$ is the $q \times q$ identity matrix. In terms of $\mathbf{S}$, the received signals are

$$
\mathbf{Y}_{i}[t]=\mathbf{S}^{q-n_{i}} \mathbf{X}[t], \quad i=1,2,
$$

where summation and multiplication are over the binary field $\mathbb{F}_{2}$. Please refer to [11] for further details.

The number of levels $n_{i}$ observed by receiver $i$ is intended to describe the SNR of the communication channel. We model a fading channel by replacing $n_{i}$ by a nonnegative random variable $N_{i}$ such that $0 \leq N_{i} \leq q$. The channel state at receiver $i$ is given by $\left\{N_{i}[t] \mid t=1,2, \ldots\right\}$, an iid random sequence with $\mathrm{PMF} P_{N_{i}}(n)$. We will assume receiver channel state information (CSI) in that $N_{i}[t]$ is known to receiver $i$ at time $t$.

As the channel states are iid, and the channels conditioned on the channel state are memoryless, we drop the symbol time index $t$ for convenience. When the transmitter signals $\mathbf{X}=\left[\begin{array}{llll}X_{1} & X_{2} & \cdots & X_{q}\end{array}\right]^{\prime}$, receiver $i$ observes

$$
\begin{aligned}
\mathbf{Y}_{i} & =\mathbf{S}^{q-N_{i}} \mathbf{X} \\
& =\left[\begin{array}{lllllll}
0 & \cdots & 0 & X_{1} & X_{2} & \cdots & X_{N_{i}}
\end{array}\right]^{\prime} .
\end{aligned}
$$

Since receiver $i$ knows the channel state $N_{i}$, receiver $i$ knows that the first $q-N_{i}$ zeroes in $\mathbf{Y}_{i}$ carry no data and that the data carrying signals are given by

$$
\left(Y_{i, q-N_{i}+1}, \ldots, Y_{q}\right)=\left(X_{1}, \ldots, X_{N_{i}}\right) .
$$


The missing signal components $X_{N_{i}+1}, \ldots, X_{q}$ have been erased by the fading channel. For convenience, we use $X^{n}$ to denote the signal vector $\left(X_{1}, \ldots, X_{n}\right)$. Thus the transmitted signal is $X=X^{q}$ and when the channel state at receiver $i$ is $N_{i}$, the receiver observation is

$$
Y_{i}=X^{N_{i}}=\left(X_{1}, \ldots, X_{N_{i}}\right)
$$

Definition 1 A q-bit layered erasure channel has input $X=X^{q} \in \mathbb{F}_{2}^{q}$, and output $Y=X^{N}$ where $N$ is an integer channel state that is independent of $X^{q}$ satisfying $P[N \geq 0]=1$ and $P[N \geq q+1]=0$.

Some useful properties of the $q$-bit layered erasure channel are gathered in the following lemma.

Lemma 1 For a q-bit layered erasure channel with output $X^{N}$ and Markov chain $V-X^{q}-X^{N}$,

(a) $I\left(X^{q} ; X^{N} \mid V\right)=H\left(X^{N} \mid V, N\right)$,

(b) $H\left(X^{N} \mid V, N\right)=\sum_{n=1}^{q} \bar{F}_{N}(n) H\left(X_{n} \mid X^{n-1}, V\right)$,

(c) $I\left(V ; X^{N}\right)=\sum_{n=1}^{q} \bar{F}_{N}(n) I\left(V ; X_{n} \mid X^{n-1}\right)$

The proof appears in the Appendix. Note that for a trivial $V$, Lemma 1)(a) implies $I\left(X^{q} ; X^{N}\right)=H\left(X^{N} \mid N\right)$.

We observe that the channel state $\mathrm{PMF} P_{N}(n)$ completely specifies a $q$-bit layered erasure channel. Given channel state PMFs $P_{N_{i}}(n)$, the broadcast channel with input $X=X^{q}$ and receiver observations $Y_{1}=X^{N_{1}}$ and $Y_{2}=X^{N_{2}}$ is described by a pair of transition probability matrices $F_{N_{1}}$ and $F_{N_{2}}$. In the parlance of [15], a $q$-bit layered erasure broadcast channel is simply a discrete memoryless $\mathrm{BC}\left(F_{N_{1}}, F_{N_{2}}\right)$. For convenience, we denote this broadcast channel simply by the tuple $\left(N_{1}, N_{2}\right)$ of channel fading random variables.

In the sequel, we will exploit the structure of the degraded broadcast channel. In the context of $q$-bit layered erasure channels, the following definition and lemma show that degradedness is associated with stochastic dominance of the $q$-bit fading distributions.

Definition 2 Random variable $N_{1}$ is stochastically larger than $N_{2}$, denoted $N_{1} \geq_{s t}$ $N_{2}$, if $\bar{F}_{N_{1}}(x) \geq \bar{F}_{N_{2}}(x)$ for all $x \geq 0$.

Lemma 2 The q-bit layered erasure broadcast channel $\left(N_{1}, N_{2}\right)$ satisfying $N_{1} \geq_{s t} N_{2}$ is a degraded broadcast channel. 


\subsection{Layered erasure broadcast channel capacity}

To identify the boundary of the capacity region $\mathcal{C}$ of the $q$-bit layered erasure broadcast channel, we start with the weighted sum rate maximization problem (3). To describe $\mathcal{C}$, we define the function

$$
\beta_{\omega}(n):=\bar{F}_{N_{1}}(n)-\omega \bar{F}_{N_{2}}(n)
$$

and construct the partition $\mathcal{I}_{1}(\omega) \cup \mathcal{I}_{2}(\omega)$ where

$$
\begin{aligned}
& \mathcal{I}_{1}(\omega)=\left\{n \mid \beta_{\omega}(n)>0\right\}, \\
& \mathcal{I}_{2}(\omega)=\left\{n \mid \beta_{\omega}(n) \leq 0\right\} .
\end{aligned}
$$

With these definitions, we can state the capacity region $\mathcal{C}$.

Theorem 2 The capacity region $\mathcal{C}$ of the $q$ bit layered erasure broadcast channel $\left(N_{1}, N_{2}\right)$ is the convex hull of the union over all $\omega \geq 0$ of rate pairs $\left(R_{1}, R_{2}\right)$ satisfying

$$
\begin{aligned}
& R_{1} \leq \sum_{n \in \mathcal{I}_{1}(\omega)} \bar{F}_{N_{1}}(n), \\
& R_{2} \leq \sum_{n \in \mathcal{I}_{2}(\omega)} \bar{F}_{N_{2}}(n) .
\end{aligned}
$$

For each $\omega \geq 0$, we present in Section 3.3 a simple scheme that achieves any weighted sum rate on the boundary of $\mathcal{C}$. In Section 3.4, we then use the method of channel enhancement to create a degraded broadcast channel that provides a matching outer bound.

\subsection{Layered Erasure BC: Achievability}

We assign signal levels $n \in \mathcal{I}_{i}(\omega)$ to user $i$. In addition, we employ independent signaling on each level. That is, $X_{1}, \ldots, X_{q}$ are iid Bernoulli $(p=1 / 2)$ random variables in each symbol period and inputs $\left\{X_{n} \mid n \in \mathcal{I}_{i}(\omega)\right\}$ are used to communicate with receiver $i$.

If level $n$ is assigned to user $i$, the level $n$ output at receiver $i$ is erased if $N_{i}<n$. That is, the erasure probability on the level $n$ subchannel is $1-\bar{F}_{N_{i}}(n)$. With independent coding on each level, the erasure channel at level $n \in \mathcal{I}_{i}(\omega)$ enables reliable communication with receiver $i$ at rate $\bar{F}_{N_{i}}(n)$. Thus

$$
\hat{R}_{i}(\omega)=\sum_{n \in \mathcal{I}_{i}(\omega)} \bar{F}_{N_{i}}(n), \quad i=1,2,
$$

is the expected number of bits addressed and delivered without erasure to receiver $i$ per symbol period. Thus this scheme enables reliable communication to the receivers at the weighted sum rate

$$
\hat{R}(\omega)=\hat{R}_{1}(\omega)+\omega \hat{R}_{2}(\omega)
$$


We note that assignment of level $n$ bits to user 1 would contribute $\bar{F}_{N_{1}}(n)$ to the weighted sum rate objective while the alternative assignment of those same bits to user 2 would contribute $\omega \bar{F}_{N_{2}}(n)$ to the objective. Hence our achievability scheme is nothing more than a simple greedy assignment policy. While this strategy is simple, it is not at all clear whether it is optimal. This policy employs independent communication on subchannel corresponding to different levels even though erasures at each receiver are correlated across these subchannels.

\subsection{Layered Erasure BC: Converse}

To verify the outer bound to the weighted sum rate, we start with the case $\omega>1$ in which we favor communication with receiver 2 . Later we will return to examine the case $\omega<1$.

Our outer bound will employ a degraded broadcast channel. We note that for arbitrary $N_{1}$ and $N_{2}$, the $q$-bit layered erasure broadcast channel is not degraded. For $\omega>1$, receiver 1 is the less favored receiver. Our approach will be to enhance the channel to this less-favored receiver by replacing the fading distribution $N_{1}$ by $\tilde{N}_{1}$ such that $\tilde{N}_{1} \geq_{\text {st }} N_{1}$. It follows that any reliable broadcast communication strategy that communicates at rates $\left(R_{1}, R_{2}\right)$ through the $q$-bit layered erasure $\mathrm{BC}\left(N_{1}, N_{2}\right)$ is also a reliable strategy through the $q$-bit fading $\mathrm{BC}\left(\tilde{N}_{1}, N_{2}\right)$, implying $\mathcal{R}\left(N_{1}, N_{2}\right) \subseteq$ $\mathcal{R}\left(\tilde{N}_{1}, N_{2}\right)$. We will show for each $\omega$ that the enhancement of the channel for the less favored receiver will not result in the transmission of additional information through that enhanced channel. Consequently, the maximum weighted sum rate $R_{1}+\omega R_{2}$ will be the same whether the maximization is over $\mathcal{R}\left(N_{1}, N_{2}\right)$ or $\mathcal{R}\left(\tilde{N}_{1}, N_{2}\right)$.

The enhanced channel for receiver 1 is given by

$$
\bar{F}_{\tilde{N}_{1}}(n)=\min \left[1, \max \left(\bar{F}_{N_{1}}(n), \omega \bar{F}_{N_{2}}(n)\right)\right]
$$

Similar to the achievability scheme which was defined in terms of $\beta_{\omega}(n)$, the converse will employ the weighted difference of complementary CDFs

$$
\tilde{\beta}_{\omega}(n):=\bar{F}_{\tilde{N}_{1}}(n)-\omega \bar{F}_{N_{2}}(n) .
$$

We note that the channel enhancement implies that

$$
\bar{F}_{\tilde{N}_{1}}(n)= \begin{cases}\bar{F}_{N_{1}}(n), & \beta_{\omega}(n)>0, \\ \min \left[1, \omega \bar{F}_{N_{2}}(n)\right], & \beta_{\omega}(n) \leq 0 .\end{cases}
$$

Taken together, (19) and 20 imply the following claim.

\section{Lemma 3}

$$
\mathcal{I}_{1}(\omega)=\left\{n \mid \beta_{\omega}(n)>0\right\}=\left\{n \mid \tilde{\beta}_{\omega}(n)>0\right\} .
$$

For $n \in \mathcal{I}_{1}(\omega), \bar{F}_{\tilde{N}_{1}}(n)=\bar{F}_{N_{1}}(n)$ and $\tilde{\beta}_{\omega}(n)=\beta_{\omega}(n)$. 
Before proceeding, we note that it is easy to verify that $\tilde{N}_{1}$ is a well-defined $q$-bit fading random variable. Next we observe that $\bar{F}_{\tilde{N}_{1}}(n) \geq \bar{F}_{N_{1}}(n)$ and thus $\tilde{N}_{1} \geq$ st $N_{1}$. Finally, we observe that one can verify that $\tilde{N}_{1} \geq_{\text {st }} N_{2}$. It then follows that $\left(\tilde{N}_{1}, N_{2}\right)$ is a degraded $q$-bit broadcast channel.

We now use Lemma 1 to apply Theorem 1 to the $q$-bit layered erasure broadcast channel $\left(\tilde{N}_{1}, N_{2}\right)$. The capacity region $\tilde{\mathcal{R}}=\mathcal{R}\left(\tilde{N}_{1}, N_{2}\right)$ is the set of all rate pairs $\left(\tilde{R}_{1}, \tilde{R}_{2}\right)$ satisfying

$$
\begin{aligned}
& \tilde{R}_{1} \leq H\left(X^{\tilde{N}_{1}} \mid \tilde{N}_{1}, V\right), \\
& \tilde{R}_{2} \leq I\left(V ; X^{N_{2}}\right)
\end{aligned}
$$

for some $\left(V, X^{q}, X^{\tilde{N}_{1}}, X^{N_{2}}\right) \in \mathcal{P}\left(\tilde{N}_{1}, N_{2}\right)$. We see that the weighted sum rate achieved by any feasible rate pair $\left(R_{1}, R_{2}\right) \in \tilde{\mathcal{R}}$ is upper bounded by the weighted sum rate

$$
\tilde{R}^{*}(\omega, V)=\tilde{R}_{1}^{*}(V)+\omega \tilde{R}_{2}^{*}(V)
$$

associated with the corner rate pair

$$
\tilde{R}_{1}^{*}(V)=H\left(X^{\tilde{N}_{1}} \mid \tilde{N}_{1}, V\right), \quad \tilde{R}_{2}^{*}(V)=I\left(V ; X^{N_{2}}\right),
$$

for some auxiliary $V$. We now identify the auxiliary $V$ that maximizes $\tilde{R}^{*}(\omega, V)$. Applying Lemma 1, we obtain the weighted rate

$$
\begin{aligned}
\tilde{R}^{*}(\omega, V) & =H\left(X^{\tilde{N}_{1}} \mid \tilde{N}_{1}, V\right)+\omega I\left(V ; X^{N_{2}}\right) \\
& =\sum_{n=1}^{q} \bar{F}_{\tilde{N}_{1}}(n) H\left(X_{n} \mid X^{n-1}, V\right)+\sum_{n=1}^{q} \omega \bar{F}_{N_{2}}(n) I\left(V ; X_{n} \mid X^{n-1}\right) \\
& =\sum_{n=1}^{q} \tilde{\beta}_{\omega}(n) H\left(X_{n} \mid X^{n-1}, V\right)+\omega \sum_{n=1}^{q} \bar{F}_{N_{2}}(n) H\left(X_{n} \mid X^{n-1}\right) .
\end{aligned}
$$

We observe that:

- The first sum in (27) is maximized if

$$
H\left(X_{n} \mid X^{n-1}, V\right)= \begin{cases}1 & \tilde{\beta}_{\omega}(n)>0 \\ 0 & \tilde{\beta}_{\omega}(n) \leq 0\end{cases}
$$

- The second sum in (27) is maximized by choosing the $X_{1}, \ldots, X_{q}$ to be iid Bernoulli $(p=1 / 2)$ random variables.

However, these two requirements are not contradictory. Given that we choose the $X_{i}$ to be iid Bernoulli $(p=1 / 2)$ random variables, we can meet the requirement (28) by choosing

$$
V=\tilde{V}=\left\{X_{n} \mid \tilde{\beta}_{\omega}(n) \leq 0\right\}
$$


In particular, we note that the role of the auxiliary is to carry the information for the favored receiver whose bits are given greater weight.

Applying the optimal $\tilde{V}$ to 27 , we see that $R^{*}(\omega)$, the maximum weighted sum rate over all feasible rate pairs $\left(R_{1}, R_{2}\right) \in \mathcal{R}\left(N_{1}, N_{2}\right)$, is upper bounded by

$$
\tilde{R}^{*}(\omega, \tilde{V})=\sum_{n: \tilde{\beta}_{\omega}(n)>0} \tilde{\beta}_{\omega}(n)+\omega \sum_{n=1}^{q} \bar{F}_{N_{2}}(n) .
$$

Applying Lemma 3, we obtain

$$
\tilde{R}^{*}(\omega, \tilde{V})=\sum_{n \in \mathcal{I}_{1}(\omega)} \bar{F}_{N_{1}}(n)+\omega \sum_{n \in \mathcal{I}_{2}(\omega)} \bar{F}_{N_{2}}(n),
$$

which is identical to $\hat{R}(\omega)$, the achievable weighted sum rate 17 . We note that this tight outer bound can also be obtained by the Körner-Marton outer bound in [15].

We now return to examine the outer bound for weight $\omega<1$. As discussed in Section 2, we can repeat the steps corresponding to equations $(22)$ through (31) with labels 1 and 2 reversed and $\omega$ replaced by $1 / \omega$. Under this role reversal, we now enhance the channel to the less-favored receiver 2 by replacing the fading distribution $N_{2}$ by $\tilde{N}_{2}$ so that the $q$-bit layered erasure BC $\left(N_{1}, \tilde{N}_{2}\right)$ has a degraded receiver 1. The maximization of the weighted sum rate for the degraded memoryless $\mathrm{BC}$ $\left(N_{1}, \tilde{N}_{2}\right)$ yields the same requirements as before: the inputs $X_{i}$ must be iid Bernoulli $(p=1 / 2)$ random variables and the auxiliary $V$ specifies the bits destined for the favored receiver, which is now receiver 1 . The corresponding bit assignment rule, derived from (14) with labels 1 and 2 reversed and $\omega$ replaced by $1 / \omega$, becomes

$$
\mathcal{I}_{1}^{\prime}(\omega)=\left\{n \mid \bar{F}_{N_{2}}(n) \leq \frac{1}{\omega} \bar{F}_{N_{1}}(n)\right\}
$$

However, trivial manipulation shows that $\mathcal{I}_{1}^{\prime}(\omega)=\mathcal{I}_{1}(\omega)$. That is, the optimal decision rule for allocating bits to each user to maximize the weighted sum rate is unchanged by the role reversal. Thus the resulting optimal weighted sum rate is still given by the achievable rate in 15 for all $\omega>0$.

\subsection{Discussion}

Theorem 2 implies that the boundary of the capacity region of the $q$ bit layered erasure broadcast channel is defined by a finite set of points. In particular at $\omega=0$, or equivalently $\omega_{1}>0$ and $\omega_{2}=0$, all bits can be assigned to receiver 1 and we obtain the extreme point $\mathbf{R}^{(0)}=\left(R_{1}^{(0)}, R_{2}^{(0)}\right)$ such that

$$
R_{1}^{(0)}=\sum_{j=1}^{q} P\left[N_{1} \geq j\right]=\mathrm{E}\left[N_{1}\right], \quad \quad R_{2}^{(0)}=0
$$




\begin{tabular}{|c|c|c|c|c|c|c|c|c|}
\hline J & $P\left[N_{1} \geq j\right]$ & $P\left[N_{2} \geq j\right]$ & $\omega_{j}$ & J & $\Omega_{j}$ & $\mathcal{I}_{1, j}$ & $\mathcal{I}_{2, j}$ & $\left(R_{1}^{(j)}, R_{2}^{(j)}\right)$ \\
\hline 1 & $3 / 4$ & $1 / 2$ & $3 / 2$ & 0 & {$[0,1 / 2]$} & $\{1,2\}$ & $\phi$ & $(1,0)$ \\
\hline 2 & $1 / 4$ & $1 / 2$ & $1 / 2$ & $\begin{array}{l}1 \\
2\end{array}$ & $\begin{array}{c}{[1 / 2,3 / 2]} \\
{[3 / 2, \infty)}\end{array}$ & $\begin{array}{c}\{1\} \\
\phi\end{array}$ & $\begin{array}{c}\{2\} \\
\{1,2\}\end{array}$ & $\begin{array}{c}(3 / 4,1 / 2) \\
(0,1)\end{array}$ \\
\hline
\end{tabular}

(a)

(b)

Table 1: Capacity region construction for the example in Equation (36).

\begin{tabular}{|c|c|c|c|c|c|c|c|c|}
\hline$j$ & $P\left[N_{1} \geq j\right]$ & $P\left[N_{2} \geq j\right]$ & $\omega_{j}$ & J & $\Omega_{j}$ & $\mathcal{I}_{1, j}$ & $\mathcal{I}_{2, j}$ & $\left(R_{1}^{(j)}, R_{2}^{(j)}\right)$ \\
\hline 1 & $3 / 4$ & $1 / 2$ & $3 / 2$ & 0 & {$[0,0]$} & $\{1,2\}$ & $\phi$ & $(3 / 4,0)$ \\
\hline 2 & 0 & $1 / 2$ & 0 & $\begin{array}{l}1 \\
2\end{array}$ & {$[0,1 / 2]$} & $\{1\}$ & $\{2\}$ & $(3 / 4,1 / 2)$ \\
\hline
\end{tabular}

(a)

(b)

Table 2: Capacity region construction for the example in Equation (37).

In addition, there is a collection of critical points $\left\{\omega_{1}, \ldots, \omega_{q}\right\}$ such that

$$
P\left[N_{1} \geq j\right]=\omega_{j} P\left[N_{2} \geq j\right], \quad j=1, \ldots, q .
$$

We define $\omega_{0}^{\prime}=0$ and $\left\{\omega_{1}^{\prime}, \omega_{2}^{\prime}, \ldots, \omega_{q^{\prime}}^{\prime}\right\}$ as the subset of unique $\omega_{j}$ arranged in strictly increasing order. With these definitions, we define the set of closed intervals $\left\{\Omega_{j} \mid j=0, \ldots, q^{\prime}\right\}$ such that

$$
\Omega_{j}= \begin{cases}{\left[\omega_{j}^{\prime}, \omega_{j+1}^{\prime}\right]} & 0 \leq j<q^{\prime}, \\ {\left[\omega_{q^{\prime}}^{\prime}, \infty\right)} & j=q^{\prime} .\end{cases}
$$

Using $\operatorname{int}\left(\Omega_{J}\right)$ to denote the interior of $\Omega_{j}$, it follows that for all $\omega \in \operatorname{int}\left(\Omega_{j}\right), \mathcal{I}_{1}(\omega)$ is unchanging; we denote this set by $\mathcal{I}_{1, j}$ and its complement by $\mathcal{I}_{2, j}$. For any $\omega \in$ $\operatorname{int}\left(\Omega_{j}\right)$, the optimal solution to the weighted sum rate maximization problem (2) is given by assigning signaling bits in $\mathcal{I}_{i, j}$ to user $i$. This solution yields an extreme point, denoted $\mathbf{R}^{(j)}=\left(R_{1}^{(j)}, R_{2}^{(j)}\right)$, of the rate region $\mathcal{R}$. When $\omega=\omega_{j}^{\prime}$ for some $j<q^{\prime}$, the weighted sum maximization is degenerate in that both extreme points $\mathbf{R}^{(j)}$ and $\mathbf{R}^{(j+1)}$ achieve maximum weighted sum rate. The full set of extreme points $\left\{\mathbf{R}^{(0)}, \ldots, \mathbf{R}^{\left(q^{\prime}\right)}\right\}$ defines the boundary of the rate region. The entire region can then be achieved by time-sharing among these extreme points.

To make this clear, consider the following example

$$
P_{N_{1}}(n)=\left\{\begin{array}{ll}
1 / 4, & n=0, \\
1 / 2, & n=1, \\
1 / 4, & n=2,
\end{array} \quad P_{N_{2}}(n)= \begin{cases}1 / 2, & n=0 \\
0, & n=1 \\
1 / 2, & n=2\end{cases}\right.
$$

in which receiver 1 has a more reliable look at the level 1 bit while receiver 2 has a better look at the level 2 bit. As a consequence, it can be shown that this $q$-bit fading 


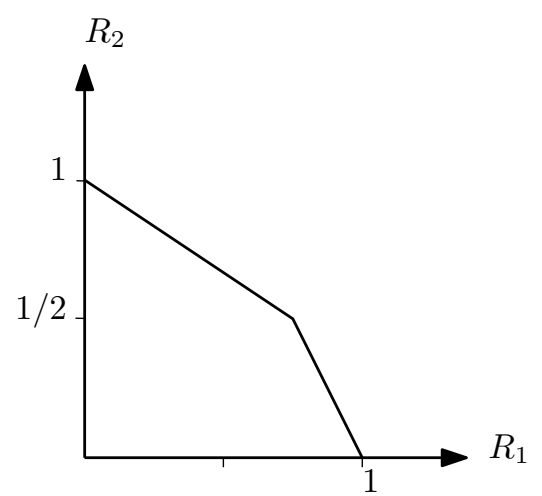

(a)

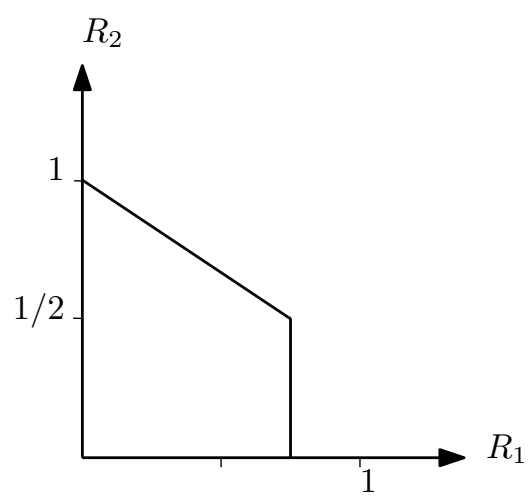

(b)

Figure 2: Examples of 2-bit capacity regions.

channel $\left(N_{1}, N_{2}\right)$ is not degraded, not less noisy, nor more capable, nor is it semideterministic. Since erasures at receiver 2 are correlated, this channel is not a parallel channel with reversely degraded components. Nevertheless, the capacity region of this channel is easy to find. From the PMFs $P_{N_{1}}\left(n_{1}\right)$ and $P_{N_{2}}\left(n_{2}\right)$, we construct the table shown in Table 1(a). This reveals $\left\{\omega_{1}^{\prime}, \omega_{2}^{\prime}\right\}=\{1 / 2,3 / 2\}$. The corresponding bit intervals, bit assignments and extreme points are shown in Table 1(b). The resulting rate region is shown in Figure 2(a).

Here is a second example with

$$
P_{N_{1}}(n)=\left\{\begin{array}{ll}
1 / 4 & n=0, \\
3 / 4 & n=1, \\
0 & n=2,
\end{array} \quad P_{N_{2}}(n)= \begin{cases}1 / 2 & n=0 \\
0 & n=1 \\
1 / 2 & n=2\end{cases}\right.
$$

From these PMFs, we construct Table 2(a), which yields $\left\{\omega_{1}^{\prime}, \omega_{2}^{\prime}\right\}=\{0,3 / 2\}$ and the intervals, bit assignments and extreme points shown in Table $2(\mathrm{~b})$. The resulting rate region is shown in Figure 2(b) where we note that in the vertical boundary by the $R_{1}$ axis arises because receiver 1 never observes bit $X_{2}$ and thus suffers no penalty when it is used by receiver 2 .

\section{Fading Gaussian BC}

Now we draw an analogy between fading Gaussian broadcast channels and the layered erasure broadcast channel and use the analogy to derive good schemes and a good outer bound for the capacity region of the fading Gaussian broadcast channel. We will show that the gap between the achievable rates of the scheme and the outer bounds are within $6.386 \mathrm{bits} / \mathrm{s} / \mathrm{Hz}$ per user of each other, irrespective of the fading processes.

For the Gaussian BC, consider fading processes such that $\left(S_{i}, \theta_{i}\right)$ is the channel state of receiver $i$, where $S_{i}$ is a real-valued non-negative channel gain and $\theta_{i} \in[0,2 \pi]$ 
is the channel phase. Random variables $S_{i}, \theta_{i}$ are assumed to be independent of each other and interpreted to be independent from symbol time to symbol time. The received signal of user $i$ at a particular time is

$$
\tilde{Y}_{i}=\sqrt{S_{i}} e^{j \theta_{i}} \tilde{X}+\tilde{Z}_{i}, \quad i=1,2,
$$

where $\tilde{X}$ is the complex baseband transmitted signal with unit power constraint and $\tilde{Z}_{i} \sim \mathcal{C} \mathcal{N}(0,1)$. The channel state $\left(S_{i}, \theta_{i}\right)$ is known at receiver $i$ but not known at the transmitter. We will refer to the fading Gaussian BC defined by (38) as the fading Gaussian BC $\left(S_{1}, S_{2}\right)$.

Now suppose user $i$ channel has phase $\theta_{i}=\theta$. Since this phase is known at the receiver, user $i$ can post-rotate its received signal by $-\theta_{i}$. We can write the complex signal input as $\tilde{X}=\left(X_{I}+j X_{Q}\right) / \sqrt{2}$ and, following post-rotation of the phase, we can represent the receiver $i$ additive noise as $\left(Z_{I i}+j Z_{Q_{i}}\right) / \sqrt{2}$, and the real and complex components $Y_{I}$ and $Y_{Q}$ of the receiver $i$ output as

$$
Y_{I i}+j Y_{Q_{i}}=\sqrt{2} e^{-j \theta_{i}} \tilde{Y}_{i}=\sqrt{S_{i}} X_{I}+Z_{I i}+j\left(\sqrt{S_{i}} X_{Q}+Z_{Q_{i}}\right), \quad i=1,2 .
$$

Thus the in-phase and quadrature channels define a pair of identical parallel fading broadcast channels, each with unit-power additive Gaussian noise. We can assume without loss of generality that signals $X_{I}$ and $X_{Q}$ are independent and each have unit power. Note this implies $\operatorname{var}[\tilde{X}]=1$. Henceforth, we will evaluate a communication scheme on the real-valued broadcast channel

$$
Y_{i}=\sqrt{S_{i}} X+Z_{i}, \quad i=1,2,
$$

that corresponds to the (post-rotated) in-phase and quadrature channels of the complex fading Gaussian BC (38). The achievable rates and outer bounds of the complex fading Gaussian BC (38) will be precisely double those obtained in the real-valued channel 40.

\subsection{Fading Gaussian BC: Outer Bound}

Let $\mathcal{C}$ be the capacity region of the real-valued fading broadcast channel 40). Fix $\omega>1$. We want to upper bound

$$
R^{*}(\omega)=\max _{\left(R_{1}, R_{2}\right) \in \mathcal{C}} R_{1}+\omega R_{2} .
$$

As in the layered erasure BC, this channel is not degraded. However, we can enhance user 1's channel to make it degraded just as we did for the binary expansion channel. The fading state of this enhanced user is denoted $\tilde{S}_{1}$ and has complementary CDF

$$
\bar{F}_{\tilde{S}_{1}}(s)=\min \left[1, \max \left(\bar{F}_{S_{1}}(s), \omega \bar{F}_{S_{2}}(s)\right)\right] .
$$

The resulting broadcast channel is now degraded with user 2 as the weaker user. As before, we now can use Theorem 1 to write

$$
R^{*}(\omega) \leq \max _{V, X} I\left(X ; Y_{1}, \tilde{S}_{1} \mid V\right)+\omega I\left(V ; Y_{2}, S_{2}\right)
$$


To characterize $R^{*}(\omega)$, we need a few definitions. When channel $i$ is in state $s$, receiver $i$ observes an output identically distributed as

$$
Y^{(s)}:=\sqrt{s} X+Z,
$$

where $Z$ denotes a $N(0,1)$ random variable identical to each $Z_{i}$. When the channel state is a random process $S$, the ergodic capacity of this point-to-point Gaussian fading channel with unit transmit power is

$$
C_{e}(S):=\int_{0}^{\infty} f_{S}(s) \frac{1}{2} \log (1+s) d s
$$

With $\bar{F}_{\tilde{S}_{1}}(s)=P\left[\tilde{S}_{1} \geq s\right]$ and $\bar{F}_{S_{2}}(s)=P\left[S_{2} \geq s\right]$ denoting the complementary CDFs of $\tilde{S}_{1}$ and $S_{2}$ respectively, we define

$$
\bar{F}_{\omega}(s)=\bar{F}_{\tilde{S}_{1}}(s)-\omega \bar{F}_{S_{2}}(s) .
$$

Finally, let $I^{\prime}\left(X ; Y^{(s)} \mid V\right)=d I\left(X ; Y^{(s)} \mid V\right) / d s$ denote the derivative of the conditional mutual information with respect to the channel SNR $s$. Standard manipulations, as shown in the appendix, yield the next claim.

\section{Lemma 4}

$$
R^{*}(\omega) \leq \max _{V, X} \int_{0}^{\infty} \bar{F}_{\omega}(s) I^{\prime}\left(X ; Y^{(s)} \mid V\right) d s+\omega C_{e}\left(S_{2}\right)
$$

Lemma 4 is the continuous-state version of Equation (27) for the layered-erasure BC. In (27), the weighted sum rate is expressed in terms of the incremental information from an improvement in channel state by one level. In Lemma $4, I^{\prime}\left(X ; Y^{(s)} \mid V\right) d s$

represents this same incremental gain. Just as in layered erasure BC, we are now able to optimize over the auxiliary $V$.

It was shown in [16] that

$$
I^{\prime}\left(X ; Y^{(s)} \mid V\right)=\frac{\log e}{2} \operatorname{mmse}(s \mid V),
$$

where, given a conditioning variable $V$,

$$
\operatorname{mmse}(s \mid V):=\mathrm{E}\left[\left(X-\mathrm{E}\left[X \mid Y^{(s)}, V\right]\right)^{2}\right]
$$

denotes the mean square error of the conditional mean estimator $\mathrm{E}\left[X \mid Y^{(s)}, V\right]$. This implies

$$
R^{*}(\omega) \leq \max _{V, X} \frac{\log e}{2} \int_{0}^{\infty} \bar{F}_{\omega}(s) \operatorname{mmse}(s \mid V) d s+\omega C_{e}\left(S_{2}\right) .
$$

We note that mmse $(s \mid V) \geq 0$ and that this minimum is achieved when $V$ specifies $X$. In addition, we also have the upper bound

$$
\operatorname{mmse}(s \mid V) \leq \frac{1}{1+s}
$$


and this upper bound is achieved when $X \sim N(0,1)$, independent of $V$. We thus obtain an upper bound to the right side of (50) when

$$
\operatorname{mmse}(s \mid V)= \begin{cases}1 /(1+s), & \bar{F}_{\omega}(s)>0 \\ 0 & \text { otherwise. }\end{cases}
$$

Defining

$$
\tilde{\mathcal{I}}_{1}(\omega)=\left\{s \geq 0 \mid \bar{F}_{\omega}(s)>0\right\}=\left\{s \geq 0 \mid \bar{F}_{\tilde{S}_{1}}(s)>\omega \bar{F}_{S_{2}}(s)\right\}
$$

and $\tilde{\mathcal{I}}_{2}(\omega)$ as its complement, we can equivalently write

$$
\operatorname{mmse}(s \mid V)= \begin{cases}1 /(1+s), & s \in \tilde{\mathcal{I}}_{1}(\omega), \\ 0 & s \in \tilde{\mathcal{I}}_{2}(\omega) .\end{cases}
$$

Applying (46) and (54) to (50) yields

$$
\begin{aligned}
R^{*}(\omega) & \leq \frac{\log e}{2} \int_{\tilde{\mathcal{I}}_{1}(\omega)} \bar{F}_{\omega}(s) \frac{1}{1+s} d s+\omega C_{e}\left(S_{2}\right) \\
& =\frac{\log e}{2}\left(\int_{\tilde{\mathcal{I}}_{1}(\omega)} \bar{F}_{\tilde{S}_{1}}(s) \frac{1}{1+s} d s+\omega \int_{\tilde{\mathcal{I}}_{2}(\omega)} \bar{F}_{S_{2}}(s) \frac{1}{1+s} d s\right) .
\end{aligned}
$$

Defining

$$
\begin{aligned}
& \mathcal{I}_{1}(\omega)=\left\{s \geq 0 \mid \bar{F}_{S_{1}}(s)>\omega \bar{F}_{S_{2}}(s)\right\}, \\
& \mathcal{I}_{2}(\omega)=\left\{s \geq 0 \mid \bar{F}_{S_{1}}(s) \leq \omega \bar{F}_{S_{2}}(s)\right\}
\end{aligned}
$$

one can verify that Lemma 3 still holds for continuous fading distributions; that is, $\tilde{\mathcal{I}}_{i}(\omega)=\mathcal{I}_{i}(\omega)$. Moreover, if $s \in \tilde{\mathcal{I}}_{1}(\omega)$, then $\bar{F}_{\tilde{S}_{1}}(s)=\bar{F}_{S_{1}}(s)$. It then follows from (56) that

$$
R^{*}(\omega) \leq \frac{\log e}{2}\left(\int_{\mathcal{I}_{1}(\omega)} \bar{F}_{S_{1}}(s) \frac{1}{1+s} d s+\omega \int_{\mathcal{I}_{2}(\omega)} \bar{F}_{S_{2}}(s) \frac{1}{1+s} d s\right) .
$$

Returning to the complex fading Gaussian BC in which rates are twice those of the in-phase channel, we observe that the components of the upper bound (58) correspond to the extreme points of the following outer bound.

Theorem 3 The capacity region of the fading Gaussian broadcast channel is contained in the convex hull of the union over all $\omega \geq 0$ of rate pairs $\left(R_{1}, R_{2}\right)$ satisfying

$$
\begin{aligned}
& R_{1} \leq R_{1}^{*}(\omega):=\log e \int_{\mathcal{I}_{1}(\omega)} \bar{F}_{S_{1}}(s) \frac{1}{1+s} d s \\
& R_{2} \leq R_{2}^{*}(\omega):=\log e \int_{\mathcal{I}_{2}(\omega)} \bar{F}_{S_{2}}(s) \frac{1}{1+s} d s
\end{aligned}
$$


We observe that for a point-to-point fading channel with unit transmit power and receiver CSI $S$, the ergodic capacity 45 can be written as

$$
C_{e}(S)=\log e \int_{0}^{\infty} \bar{F}_{S}(s) \frac{1}{1+s} d s .
$$

When $I_{j}(\omega)$ is empty and all channel states are "assigned" to receiver $i \neq j$, we see that the outer bound for $R_{i}$ is simply the ergodic capacity of the point-to-point fading channel to receiver $i$. Thus the outer bound rates (59) are tight when the channel input is assigned to a single receiver. However, when there is a partitioning by channel state of the available ergodic capacity, we will see that the outer bound rates $(59)$ are loose in the absence of an achievability scheme in which an auxiliary $V$ satisfies (54).

\subsection{Fading Gaussian BC: Achievability}

We will employ an achievable scheme based on superposition of independent binary streams composed of \pm 1 symbols. In particular, for the real-valued fading Gaussian BC (40), let the channel input $X$ at time $t$ be given by

$$
X[t]=\sqrt{3} \sum_{n=1}^{\infty} \tilde{X}_{n}[t] 2^{-n}
$$

where $\tilde{X}_{1}[t], \tilde{X}_{2}[t], \ldots$ are independent signals taking values in $\{-1,1\}$ equiprobably. Each stream $\left\{\tilde{X}_{n}[t]\right\}$ will communicate an independent layer $n$ data stream, encoded at a rate $r_{n}$ which is chosen to tolerate interference from the other streams, channel variations and receiver noise. Note that unlike the rest of the paper, we make explicit the dependency on the symbol time $t$ to emphasize that the symbols within a layer are coded across time.

Each receiver will use a two-stage decoding procedure. First, receiver $i$ employs a detector to form estimates $\hat{X}_{1}[t], \hat{X}_{2}[t], \ldots$ over a single-symbol period of the antipodal binary symbols $\tilde{X}_{1}[t], \tilde{X}_{2}[t], \ldots$ at each time instant $t$. In the second stage, receiver $i$ employs the estimates $\hat{X}_{n}[1], \hat{X}_{n}[2], \ldots$ to decode the coded sequence $\tilde{X}_{n}[1], \tilde{X}_{n}[2], \ldots$ for each layer $n$ stream that the receiver is assigned.

As the channel state $S_{i}$ is known at receiver $i$, the receiver outputs can be normalized by the channel gains, so that the real broadcast channel (40) with input $X[t]$ given by (61) is equivalent to

$$
\tilde{Y}_{i}[t]=\sum_{n=1}^{\infty} \tilde{X}_{n}[t] 2^{-n}+\frac{Z_{i}[t]}{\sqrt{3 S_{i}}}, \quad i=1,2 .
$$

In the broadcast channel, different layers are assigned to different users. The code rate employed on a layer depends on which receiver is assigned that layer, which in turn depends on the channel state distributions $\bar{F}_{S_{i}}(s)$ and the weight factor $\omega$. 


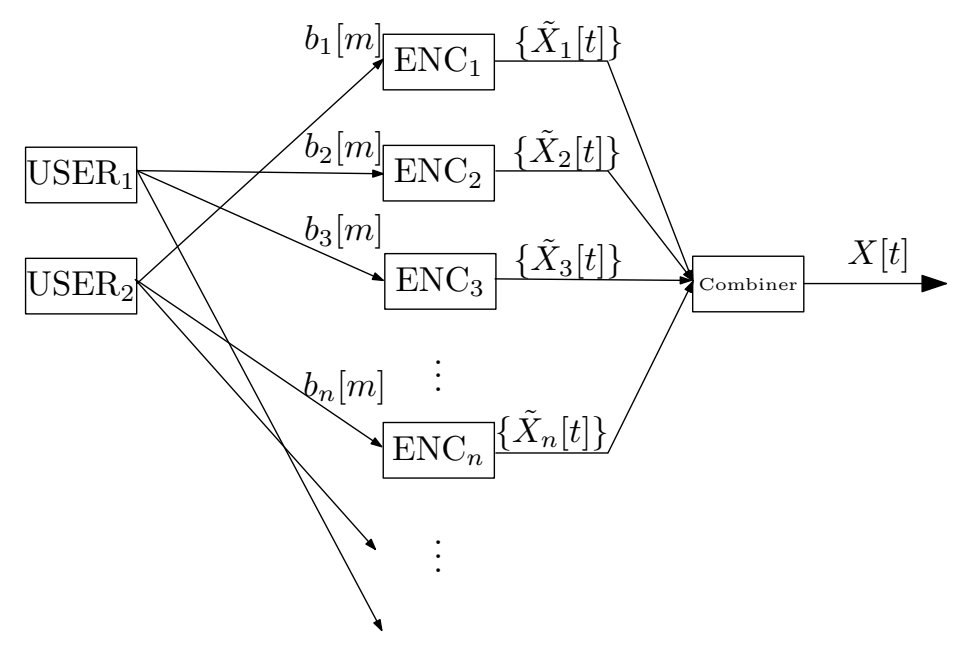

Figure 3: In this example of the in-phase baseband transmitter, the layer 2 and layer 3 signals are assigned to user 1 while the layer 1 signal is assigned to user 2 . For signal layer $k$ assigned to user $i$, the bit stream $b_{k}[n]$ is sent to the layer $k$ encoder at rate $r_{k, i}$. The transmitted signal $X[t]$ is given by Equation (61).

Before proceeding to the analysis of this system, we develop some terminology to describe our signaling scheme. We start by expressing the number $b \in[0,1]$ as the binary expansion $b=\sum_{n=1}^{\infty} b_{n} 2^{-n}$ with each $b_{n} \in\{0,1\}$. We denote such a binary expansion by $b=\left\langle b_{1}, b_{2}, \ldots\right\rangle_{\{0,1\}}$, where the subscript $\{0,1\}$ denotes the range of each element. Next we observe $a=2 b-1$ spans the interval $[-1,1]$ and has the corresponding expansion

$$
a=2 b-1=2 \sum_{n=1}^{\infty} b_{n} 2^{-n}-\sum_{n=1}^{\infty} 2^{-n}=\sum_{n=1}^{\infty}\left(2 b_{n}-1\right) 2^{-n} .
$$

Defining $a_{n}=2 b_{n}-1$, we see that any $a \in[-1,1]$ can be expressed in terms of the antipodal expansion $a=\sum_{n=1}^{\infty} a_{n} 2^{-n}$ with each $a_{n} \in\{-1,1\}$. Moreover, given $a \in[-1,1]$, we can construct the corresponding antipodal expansion, denoted $\left\langle a_{1}, a_{2}, \ldots\right\rangle_{\{ \pm 1\}}$, via $a_{1}=\operatorname{sgn}(a), a_{2}=\operatorname{sgn}\left(a-a_{1} / 2\right)$, and

$$
a_{n+1}=\operatorname{sgn}\left(a-\sum_{j=1}^{n} a_{j} 2^{-j}\right)
$$

In certain situations, we will employ the signaling scheme (61), but with only a finite number $m$ of signal levels. In this case, the transmitted signal constellation for a single symbol period is given by

$$
\mathcal{X}=\left\{\sum_{n=1}^{m} x_{n} 2^{-n} \mid x_{n} \in\{-1,1\}, n=1,2, \ldots, m\right\} .
$$

That is, $\mathcal{X}$ consists of the set of all $m$-bit antipodal expansions $\left\langle x_{1}, \ldots, x_{m}\right\rangle_{\{ \pm 1\}}$. In this case, a receiver may observe $y \in[-1,1]$ and wish to determine the $\hat{x} \in \mathcal{X}$ closest 
to $y$. Expressing $y$ in terms of its antipodal expansion $y=\sum_{n=1}^{\infty} y_{n} 2^{-n}$, one can show that the closest $\hat{x} \in \mathcal{X}$ is $\hat{x}=\sum_{n=1}^{m} y_{n} 2^{-n}$, which is simply the truncation of the antipodal expansion of $y$ to its first $m$ bits. We refer to this as the truncation property of the antipodal expansion 17

Returning to the real-valued fading Gaussian BC 62 , consider a receiver for $\tilde{Y}[t]$, which could be either $\tilde{Y}_{i}[t]$ with channel state $S_{i}[t]=s$. To evaluate this receiver, we drop the time index $t$ and consider the first stage detection over a single symbol period with channel state $S_{i}=s$. The received signal is

$$
\tilde{Y}=\tilde{X}+\tilde{Z}
$$

where the transmitted signal constellation is the set of all $\tilde{X}=\sum_{n=1}^{\infty} \tilde{X}_{n} 2^{-n} \in[-1,1]$ and the receiver noise is $\tilde{Z}=Z / \sqrt{3 s}$. For this receiver, the signal set consists of all antipodal expansions $\tilde{X}=\left\langle\tilde{X}_{1}, \tilde{X}_{2}, \ldots\right\rangle_{\{ \pm 1\}} \in[-1,1]$. The receiver detects the input bits $\left\{\tilde{X}_{i}\right\}$ by mapping the observation $\tilde{Y}$ to the nearest signal constellation point

$$
\hat{X}=\left\langle\hat{X}_{1}, \hat{X}_{2}, \ldots\right\rangle_{\{ \pm 1\}}=\sum_{n=1}^{\infty} \hat{X}_{n} 2^{-n}
$$

where $\hat{X}_{n} \in\{ \pm 1\}$ is the receiver's best estimate of the layer $n$ input bit $\tilde{X}_{n}$. This minimum distance detection reduces to

$$
\hat{X}=\max (-1, \min [1, \tilde{Y}]) .
$$

such that given $\hat{X}$, we derive the antipodal bits $\left\{\hat{X}_{n}\right\}$ using the method of 64. That is, when $\tilde{Y} \in[-1,1], \hat{X}=\tilde{Y}$ and the binary expansion of $\tilde{Y}$ is our best estimate for the bits $\left\{\tilde{X}_{n}\right\}$. When $\tilde{Y}>1$, the detector estimates $\hat{X}_{n}=1$ for all $n$ and when $\tilde{Y}<-1$, the detector estimate $\hat{X}_{n}=-1$ for all $n$.

As a notation convenience, we use $\tilde{X}^{n}$ to represent the $n$-bit antipodal expansion $\left\langle\tilde{X}_{1}, \ldots, \tilde{X}_{n}\right\rangle_{\{ \pm 1\}}=\sum_{j=1}^{n} \tilde{X}_{j} 2^{-j}$. This notation is also applied to $\hat{X}^{n}, \tilde{Y}^{n}$ and sample values such as $\tilde{x}^{n}$ of $\tilde{X}^{n}$. For the detector (68), we observe that the probability $P\left[\hat{X}_{n}=\tilde{X}_{n}\right]$ of a correct bit decision at layer $n$ is lower bounded by

$$
P\left[\hat{X}_{n}=\tilde{X}_{n}\right] \geq P\left[\hat{X}^{n}=\tilde{X}^{n}\right] .
$$

To proceed, we will find a lower bound to $P\left[\hat{X}^{n}=\tilde{x}^{n} \mid \tilde{X}^{n}=\tilde{x}^{n}\right]$ that holds for all $\tilde{x}^{n}$. Given $\tilde{X}^{n}=\tilde{x}^{n}$, we can write $\tilde{X}=\tilde{x}^{n}+U_{n}$, where

$$
U_{n}=\sum_{j=n+1}^{\infty} \tilde{X}_{j} 2^{-j}
$$

\footnotetext{
${ }^{1}$ We observe that the truncation property is not shared by the ordinary binary expansion. For example, $15 / 32$ has the binary expansion $\langle 0,1,1,1,1,0, \ldots\rangle_{\{0,1\}}$ but $\langle 1,0\rangle_{\{0,1\}}=1 / 2$ and $\langle 1,0,0\rangle_{\{0,1\}}$ are the 2-bit and 3-bit binary expansions closest to 15/32. However, 15/32 has the antipodal expansion $\langle 1,-1,1,1,1\rangle_{\{ \pm 1\}}$ and $\langle 1,-1\rangle_{\{ \pm 1\}}$ and $\langle 1,-1,1\rangle_{\{ \pm 1\}}$ are the closest 2-bit and 3-bit antipodal expansions.
} 
is a continuous uniform $\left(-2^{-n}, 2^{-n}\right)$ random variable that is independent of $\tilde{X}^{n}$ and $\tilde{Z}$. We refer to $U_{n}$ as $L S B$ interference since at level $n$ it is the superposition of signals for the less significant bits $\left\{\tilde{X}_{j} \mid j>n\right\}$.

Thus, given $\tilde{X}^{n}=\tilde{x}^{n}$, the receiver observes

$$
\tilde{Y}=\tilde{x}^{n}+U_{n}+\tilde{Z} .
$$

From $(68)$ and the truncation property of the antipodal expansion, $\left|\tilde{Y}-\tilde{x}^{n}\right|<2^{-n}$ implies $X^{n}=\tilde{x}^{n}$. With the definition of the conditional probability

$$
P\left[\tilde{x}^{n}-2^{-n} \leq \tilde{Y}<\tilde{x}^{n}+2^{-n} \mid \tilde{X}^{n}=\tilde{x}^{n}\right]
$$

it follows that

$$
P\left[\hat{X}^{n}=\tilde{x}^{n} \mid \tilde{X}^{n}=\tilde{x}^{n}\right] \geq P\left[C\left(\tilde{x}^{n}\right)\right] .
$$

From 71 , (72) and independence of $\tilde{X}^{n}, U_{n}$, and $\tilde{Z}$,

$$
P\left[C\left(\tilde{x}^{n}\right)\right]=P\left[-2^{-n} \leq U_{n}+\tilde{Z}<2^{-n}\right],
$$

which is independent of $\tilde{x}^{n}$.

When the fading state is reasonable, the lower bound $P\left[C\left(\tilde{x}^{n}\right)\right]$ is sufficient to characterize the reliability of the bit detection at layer $n$. However, as the fading state becomes too weak, this lower bound will approach zero, corresponding to our detector converging to random guessing. Analysis of the detector for weak fading states is complex but, fortunately, unnecessary. As the channel state $S=s$ is known at the receiver, we can simplify our analysis by assuming the detector sets a threshold $\hat{n}(s)$ such that for layers $n>\hat{n}(s)$ the receiver simply sets $\hat{X}_{n}$ to a random guess. To be precise, a receiver with observation $\tilde{Y}$ in channel state $S=s$ implements the first stage detector

$$
\begin{aligned}
\left\langle\hat{Y}_{1}, \hat{Y}_{2}, \ldots\right\rangle_{\{ \pm 1\}} & =\max (-1, \min [1, \tilde{Y}]), \\
\hat{X}_{n} & = \begin{cases}\hat{Y}_{n} & 1 \leq n \leq \hat{n}(s), \\
W_{n} & n>\hat{n}(s),\end{cases}
\end{aligned}
$$

where $W_{1}, W_{2}, \ldots$ is simply an iid sequence of equiprobable Bernoulli random variables, independent of any observations. We choose the threshold $\hat{n}(s)$ as the largest $n$ such that $P\left[C\left(\tilde{x}^{n}\right)\right] \geq 1 / 2$. This provides a simple guarantee that our detector never does worse than random guessing.

The exact calculation of $P\left[C\left(\tilde{x}^{n}\right)\right]$ is shown in a generalized form in the proof of Lemma 6. We will express the result in terms of

$$
\begin{aligned}
a_{n}(s) & :=3 s 2^{-2 n} \\
G(x) & :=x Q(x)-\frac{1}{\sqrt{2 \pi}} e^{-x^{2} / 2} \\
\epsilon_{d}(a) & :=\frac{G\left(\sqrt{a}\left(1+2^{-d}\right)\right)-G\left(\sqrt{a}\left(1-2^{-d}\right)\right)}{\sqrt{a} 2^{-d}}
\end{aligned}
$$


and

$$
\hat{\epsilon}_{d}(a):=\min \left[1 / 2, \epsilon_{d}(a)\right] .
$$

We note that $a_{n}(s)$ is the SNR of bit $\tilde{X}_{n}$ under channel state $s$ and that $G(x)$ is simply the integral of $Q(x)$. In the proof of Lemma 6, we show $1-P\left[C\left(\tilde{x}^{n}\right)\right]=\epsilon_{0}\left(a_{n}(s)\right)$. By using random guessing for bits $\left\{\tilde{X}_{n} \mid n>\hat{n}(s)\right\}$, the next lemma follows.

Lemma 5 In channel state $S=s$, the bit detector 75 yields a BSC from $\tilde{X}_{n}$ to $\hat{X}_{n}$ with crossover probability $p_{n, 0}(s) \leq \hat{\epsilon}_{0}\left(a_{n}(s)\right)$.

This is the $d=0$ special case of Lemma 6 in Section 4.3. It is straightforward to verify that $\epsilon_{0}(a)=[G(2 \sqrt{a})-G(0)] / \sqrt{a}$ is decreasing in $a$ and that $\epsilon_{0}(a=0.5405)=1 / 2$. The upper bound of $1 / 2$ inherent in the definition of $\hat{\epsilon}_{0}(a)$ comes from defining the threshold

$$
\hat{n}(s)=\max \left\{n \mid \epsilon_{0}\left(a_{n}(s)\right) \leq 1 / 2\right\} \approx\left\lfloor 0.349+\frac{1}{2} \log s\right\rfloor .
$$

The threshold $\hat{n}(s)$ is chosen simply so that for bits at level $n \leq \hat{n}(s)$, the effective channel from $\tilde{X}_{n}$ to the receiver guess $\hat{X}_{n}$ is a binary symmetric channel (BSC) with crossover probability less than $1 / 2$.

The implication of Lemma 5 is that receiver $i$ observes level $n$ bits through a BSC with time-varying crossover probability $p_{n, 0}\left(S_{i}\right)$. We employ this signaling and detection scheme on both the in-phase and quadrature channels, coupled with coding over time. With $H(p)$ denoting the binary entropy function, user $i$ can communicate reliably using the level $n$ channel at rate

$$
r_{n}^{(i)} \geq 2 \mathrm{E}_{S_{i}}\left[1-H\left(p_{n, 0}\left(S_{i}\right)\right)\right] \geq 2 \mathrm{E}_{S_{i}}\left[1-H\left(\hat{\epsilon}_{0}\left(a_{n}\left(S_{i}\right)\right)\right)\right]
$$

For convenience, we define

$$
\hat{H}_{d}(s):=1-H\left(\hat{\epsilon}_{d}(s)\right) .
$$

In our achievability scheme, we are free to assign each signal level $n$ arbitrarily. By considering the assignments of signal levels $n \in \hat{\mathcal{N}}_{i}$ to each receiver, we can achieve the following rate region.

Theorem 4 The capacity region of the fading Gaussian $B C\left(S_{1}, S_{2}\right)$ includes all rate pairs $\left(R_{1}, R_{2}\right)$ satisfying

$$
R_{i} \leq 2 \sum_{n \in \hat{\mathcal{N}}_{i}} E_{S_{i}}\left[\hat{H}_{0}\left(3 S_{i} 2^{-2 n}\right)\right], \quad i=1,2,
$$

for some partition $\hat{\mathcal{N}}_{1} \cup \hat{\mathcal{N}}_{2}$ of $\{1,2, \ldots\}$.

In Section 5, we show that the achievable rates of Theorem 4 are within a constant gap of the Theorem 3 outer bound. However, Theorem 4 does not exploit knowledge of the channel fading distributions. In the following subsection, we describe how a receiver can offer a considerable improvement in rates for specific channel state distributions. 


\subsection{Improved Rates via Reverse Stripping}

While the binary expansion superposition achievability scheme in Section 4.2 mimics the structure of the layered erasure channel, it has two disadvantages:

- At level $n$, the detector makes a hard decision on the bits at levels 1 through $n$. These decisions do not exploit coding over time.

- The hard decisions at level $n$ are subject to LSB interference from bits at levels $k>n$.

A consequence of the detector's hard decisions is that binary expansion superposition sacrifices low SNR performance. In particular, 80 shows that the level $n$ bit is useless for channels state $s<2^{2(n-0.349)}$, implying no channel state $s \leq 2^{1.302}$ communicates information. While we are perhaps stuck with the hard decisions, the LSB interference can be mitigated in some circumstances. To explain this, we suppose initially that the weight $\omega$ is chosen so that all layers are assigned to user $i$. Although user $i$ could obtain his point-to-point ergodic capacity rate via constant power Gaussian codes, it's instructive to examine what can be achieved with the binary expansion superposition scheme. In this case, we could do the following reverse stripping:

- Select a level $n_{\max }$, corresponding to the least significant bit we will transmit, so that the forfeited capacity is negligible.

- At layer $n=n_{\max }$, make hard decisions $\tilde{X}_{n}[t]$ using the BES detector 75 . In this case, the layer $n_{\max }$ crossover probability is reduced due to the absence of LSB interference.

- After a code block has been sent, decode the level $n$ codeword and then strip the level $n$ signal from the received signal.

- Go back and redo the binary detection for level $n-1$ using the residual received signal. Now detection at level $n-1$ is no longer subject to LSB interference from level $n$.

- Repeat this process all the way down to bit $X_{1}$.

We call this process reverse stripping because we decode and strip the signals in the order of increasing signal power, which is the reverse of the decoding order for Gaussian superposition codes.

At each level $n$, this process reduces the crossover probability for the binary detector 75 for a given channel state $S_{i}=s$. In particular, in the error probability analysis of equations 66 through 74 , the received signal is still $\tilde{Y}=\tilde{X}+\tilde{Z}$, but the transmitted signal is now a constellation point

$$
\tilde{X}=\left\langle\tilde{X}_{1}, \ldots, \tilde{X}_{n_{\max }}\right\rangle_{\{ \pm 1\}}=\sum_{j=1}^{n_{\max }} \tilde{X}_{j} 2^{-j}
$$


For detection of bit $n=n_{\max },(69)$ and (72) remain unchanged; however, given $\tilde{X}^{n}=\tilde{x}^{n}$, we now have $\tilde{Y}=\tilde{x}^{n}+Z$. That is, the LSB interference $U_{n}$ is now zero. Following (74), the probability of correct detection of the bit $X_{n}$ for $n=n_{\max }$ is lower bounded by

$$
P\left[C\left(\tilde{x}^{n}\right)\right]=P\left[-2^{-n} \leq \tilde{Z}<2^{-n}\right]=1-2 Q\left(\sqrt{a_{n}(s)}\right) .
$$

We note from 78 that

$$
\epsilon_{\infty}(a):=\lim _{d \rightarrow \infty} \epsilon_{d}(a)=\left.2 \frac{d G(x)}{d x}\right|_{x=\sqrt{a}}=2 Q(\sqrt{a}) .
$$

In addition, we define $\hat{\epsilon}_{\infty}(a)=\min \left[1 / 2, \epsilon_{\infty}(a)\right]$. Denoting the crossover probability at level $n$ by $p_{n, \infty}(s), 84$ and 85 yield the upper bound

$$
p_{n, \infty}(s) \leq 1-P\left[C\left(\tilde{x}^{n}\right)\right]=\hat{\epsilon}_{\infty}\left(a_{n}(s)\right)
$$

Following the reverse stripping strategy, we strip the coded signal $\tilde{X}_{n_{\max }}[t]$. The residual transmitted signal is now $\tilde{X}=\sum_{n=1}^{n_{\max }-1} \tilde{X}_{n} 2^{-n}$. Repeating the same analysis, the error probability for bit $n=n_{\max }-1$ also satisfies the upper bound (86). By successive reverse stripping, the upper bound 86 holds for all bits $\tilde{X}_{n}$. It follows that user $i$ achieves the ergodic rate

$$
R_{i}=2 \sum_{n=1}^{\infty} \mathrm{E}_{S_{i}}\left[\hat{H}_{\infty}\left(3 S_{i} 2^{-2 n}\right)\right] .
$$

Reverse stripping also can be exploited for binary expansion superposition signaling in the general setting when the bits are assigned to different users. Here suppose that user $i$ is assigned bit levels

$$
\mathcal{N}_{i}=\bigcup_{j=1}^{k}\left\{n_{j}, n_{j}+1, \ldots, l_{j}\right\}
$$

where $n_{j} \leq l_{j}<n_{j+1}$. In this case, we can apply reverse stripping to decoding each interval of bits $\left\{\tilde{X}_{n_{j}}, \ldots, \tilde{X}_{l_{j}}\right\}$. Starting with bit $n=l_{j}$, the probability of correct decoding of bit $\tilde{X}_{n}$ is lower bounded by

$$
P\left[C\left(\tilde{x}^{n}\right)\right]=P\left[\tilde{Y}^{n}=\tilde{x}^{n} \mid \tilde{X}^{n}=\tilde{x}^{n}\right] .
$$

Given $\tilde{X}^{n}=\tilde{x}^{n}$, we can write $\tilde{X}=\tilde{x}^{n}+U_{n}$ with

$$
U_{n}=\sum_{j=n+1}^{\infty} \tilde{X}_{j} 2^{-j}
$$

denoting the LSB interference. In fact, user $i$ will have previously decoded (via reverse stripping) the LSBs $\cup_{k=j+1}^{\infty}\left\{\tilde{X}_{n_{k}}, \ldots, \tilde{X}_{l_{k}}\right\}$ and these bits can be stripped from the 
LSB interference $U_{n}$. However, $U_{n}$ also contains bits $\left\{X_{n+1} \ldots, X_{n_{j+1}-1}\right\}$ that are assigned to the other user and these bits may be encoded at a rate that user $i$ cannot decode and strip reliably. Consequently, we assume that these bits are undecodable by user $i$. Moreoever, as these same bits that are the most significant bits in the LSB interference, they dominate the LSB interference. Hence, there is only a small penalty in the assumption that the LSB interference $U_{n}$ in (89) contains no known bits and is thus statistically identical to a continuous uniform $\left(-2^{-n}, 2^{-n}\right)$ random variable. To be precise, we obtain a lower bound to the probability of correct decoding because the LSB interference $U_{n}$ with some known bits $\tilde{X}_{j}$ can be degraded by replacing those known (and stripped) bits with antipodal \pm 1 noise to create the continuous uniform $U_{n}$. With this assumption, analysis of the detection of bit $n=l_{j}$ is identical to that described in equations (69) through (74) and the crossover probability is given by Lemma 5 .

Now suppose in the reverse stripping process we are at level $n=l_{j}-d$ and we wish to detect bit $\tilde{X}_{n}=\tilde{X}_{l_{j}-d}$ having already decoded and stripped bits $\tilde{X}_{l_{j}-d+1}$ through $\tilde{X}_{l_{j}}$. In this case, given $\tilde{X}^{n}=\tilde{x}^{n}$, the residual received signal is

$$
\tilde{Y}=\tilde{x}^{n}+U_{n}+\tilde{Z} \text {. }
$$

The residual LSB interference, after stripping the LSB interference at levels $l_{j}$ through $l_{j-d+1}$, is

$$
U_{n}=\sum_{k=l_{j}+1}^{\infty} \tilde{X}_{k} 2^{-k}=\sum_{k=n+d+1}^{\infty} \tilde{X}_{k} 2^{-k}=2^{-d} \tilde{U}_{n}
$$

where

$$
\tilde{U}_{n}=\sum_{k^{\prime}=n+1}^{\infty} \tilde{X}_{k^{\prime}+d} 2^{-k^{\prime}}
$$

With respect to the detection of bit $\tilde{X}_{n}$ for user $i$, the most significant bits in $\tilde{U}_{n}$ are assigned to the other user and thus cannot be assumed to be decodable by user $i$. Hence we can lower bound the probability of correct detection by making the worst-case assumption that $\tilde{U}_{n}$ is a continuous uniform $\left(-2^{-n}, 2^{-n}\right)$ random variable. Nevertheless, as $\tilde{U}_{n}$ is identical to the LSB interference at layer $n$ without reverse stripping, we say that the LSB interference $U_{n}=2^{-d} \tilde{U}_{n}$ is at depth $d$. In this case, the probability of correct detection at level $n$ with LSB interference at depth $d$ is lower bounded by

$$
\begin{aligned}
P\left[C\left(\tilde{x}^{n}\right)\right] & =P\left[\tilde{x}^{n}-2^{-n} \leq \tilde{Y}<\tilde{x}^{n}+2^{-n} \mid \tilde{X}^{n}=\tilde{x}^{n}\right] \\
& =P\left[-2^{-n} \leq 2^{-d} \tilde{U}_{n}+\tilde{Z}<2^{-n}\right]
\end{aligned}
$$

In the proof of the following Lemma, we show that $1-P\left[C\left(\tilde{x}^{n}\right)\right]=\epsilon_{d}\left(a_{n}(s)\right)$. In channel state $s$, we guess bits $\hat{X}_{n}$ for layers $n>\hat{n}(s)$, yielding an upper bound of $\hat{\epsilon}_{d}\left(a_{n}(s)\right)$ for the crossover probability at all layers $n$.

Lemma 6 In channel state $S=s$ with LSB interference at depth $d$, the bit detector 75) yields a BSC from $\tilde{X}_{n}$ to $\hat{X}_{n}$ with crossover probability $p_{n, d}(s) \leq \hat{\epsilon}_{d}\left(a_{n}(s)\right)$. 


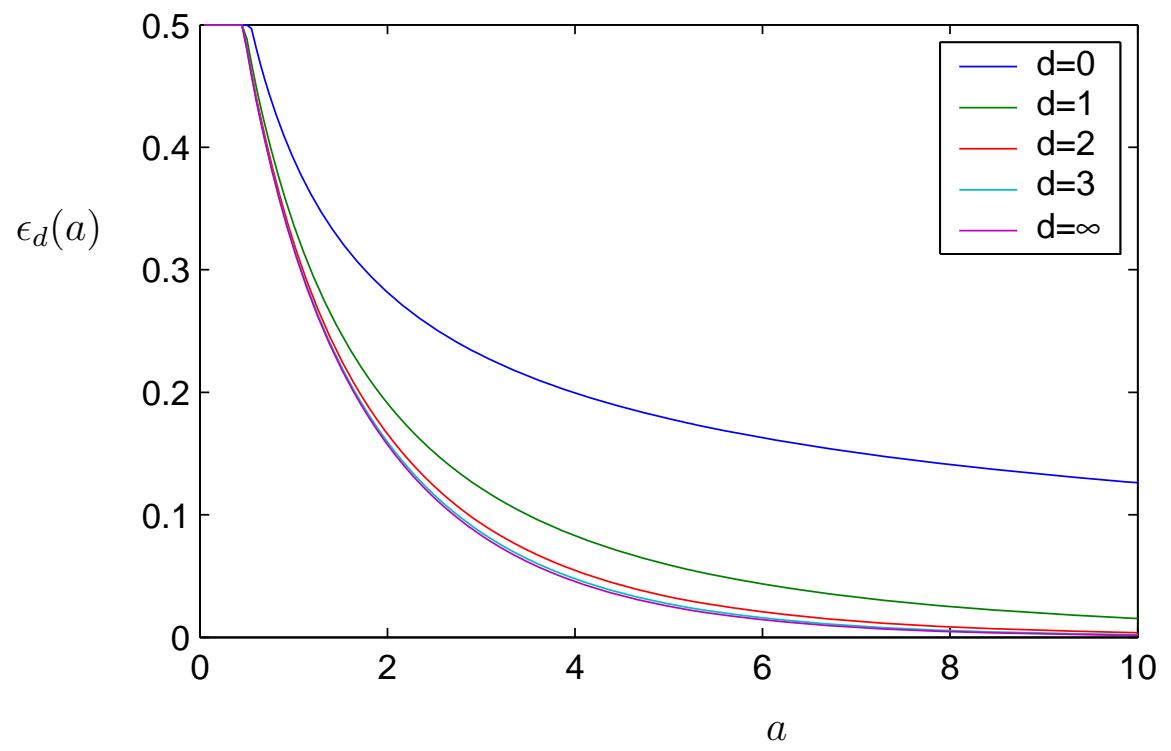

Figure 4: In channel state $s$, the probability of bit error for the BES detector at level $n$ with LSB interference at depth $d$ is $\hat{\epsilon}_{d}(a)$ for $a=a_{n}(s)=3 s 2^{-2 n}$.

We note that the crossover probability of Lemma 5 is the special case of LSB interference at depth $d=0$. Similarly, when all bits are assigned to user $i$ and there is no LSB interference, the crossover probability $\hat{\epsilon}_{\infty}\left(a_{n}(s)\right)$ given in 86 corresponds to LSB interference at depth $d \rightarrow \infty$. In Figure 4, we plot $\hat{\epsilon}_{d}(a)$ and $\hat{\epsilon}_{\infty}(a)$. We see there is a considerable improvement in detection when we go from LSB interference at depth $d=0$ to $d=1$ and that LSB interference at depth $d \geq 3$ is essentially indistinguishable from no LSB interference.

We now apply Lemma 6. Given a weight factor $\omega$, we assume that bits $n \in \mathcal{N}_{i}$ given by (87) are assigned to user $i$. At each level $n$, the interference must overcome LSB interference at a depth $d_{n}$ that is specified by the assignments $\mathcal{N}_{i}$. In particular, if $n \in N_{i}$, then

$$
d_{n}=\min \left\{d \geq 0 \mid n+d+1 \notin N_{i}\right\} .
$$

With reverse stripping, receiver $i$ observes the bits of layer $n \in N_{i}$ through a BSC with time-varying crossover probability $p_{n, d_{n}}\left(S_{i}\right)$, enabling reliable communication at rate

$$
r_{n}^{(i)} \geq 2 \mathrm{E}_{S_{i}}\left[1-H\left(p_{n, d_{n}}\left(S_{i}\right)\right)\right]
$$

By partitioning the signal levels, we obtain the following achievable rate region.

Theorem 5 The capacity region of the fading Gaussian $B C\left(S_{1}, S_{2}\right)$ includes all rate 
pairs $\left(R_{1}, R_{2}\right)$ satisfying

$$
R_{i} \leq 2 \sum_{n \in \hat{\mathcal{N}}_{i}} E_{S_{i}}\left[\hat{H}_{d_{n}}\left(3 S_{i} 2^{-2 n}\right)\right], \quad i=1,2,
$$

for some partition $\hat{\mathcal{N}}_{1} \cup \hat{\mathcal{N}}_{2}$ of $\{1,2, \ldots\}$.

\subsection{Examples}

In this section, we compare achievable rates and the outer bound of Theorem 3 for some simple examples. Beyond an initial degraded AWGN BC example, the subsequent examples consider non-degraded broadcast channels which, by construction, exhibit signficant gains over time sharing. Our approach is to evaluate the outer bound of Theorem 3 and use the associated partition of signal levels to guide the assignment of BES bit levels in the achievability scheme.

\section{Intermittent AWGN channels}

First we consider channels in which each user $i$ has channel state $S_{i}$ described by

$$
\bar{F}_{S_{i}}(s)= \begin{cases}1 & s<0 \\ p_{i} & 0 \leq s \leq s_{i}^{*}, \\ 0 & s>s_{i}^{*} .\end{cases}
$$

That is, each user $i$ has an intermittent $A W G N$ channel in which the SNR is $s_{i}^{*}$ with probability $p_{i}$ and is otherwise zero. We refer to $p_{i}$ as the channel activity factor and $s_{i}^{*}$ as the maximum SNR. In all such examples, we assume without loss of generality that $s_{2}^{*} \leq s_{1}^{*}$. We make the further assumption that $p_{2} \geq p_{1}$. That is, the intermittent good channel of user 2 is less good than that of user 1 but user 2 more often has a good channel. It will be convenient to express our results in terms of the ratio $\rho=p_{1} / p_{2}$ and the ergodic capacity

$$
C_{i}=p_{i} \log \left(1+s_{1}^{*}\right)
$$

that user $i$ would obtain with the full devotion of the transmitter's resources.

For the outer bound, the partition (57) yields

$$
\begin{aligned}
& \mathcal{I}_{1}(\omega)= \begin{cases}{\left[0, s_{1}^{*}\right]} & 0 \leq \omega<\rho, \\
\left(s_{2}^{*}, s_{1}^{*}\right] & \rho \leq \omega,\end{cases} \\
& \mathcal{I}_{2}(\omega)= \begin{cases}\left(s_{1}^{*}, \infty\right) & 0 \leq \omega<\rho, \\
{\left[0, s_{2}^{*}\right] \cup\left(s_{1}^{*}, \infty\right)} & \rho \leq \omega .\end{cases}
\end{aligned}
$$

It follows from Theorem 3 that the outer bound region has extreme points

$$
\begin{aligned}
& R_{1}^{*}(\omega)= \begin{cases}C_{1} & 0 \leq \omega<\rho, \\
C_{1}-\rho C_{2} & \rho \leq \omega,\end{cases} \\
& R_{2}^{*}(\omega)= \begin{cases}0 & 0 \leq \omega<1, \\
C_{2} & 1 \leq \omega .\end{cases}
\end{aligned}
$$




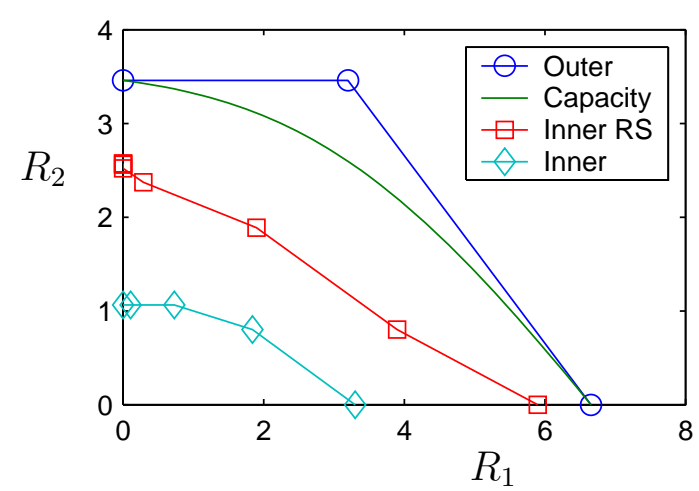

(a)

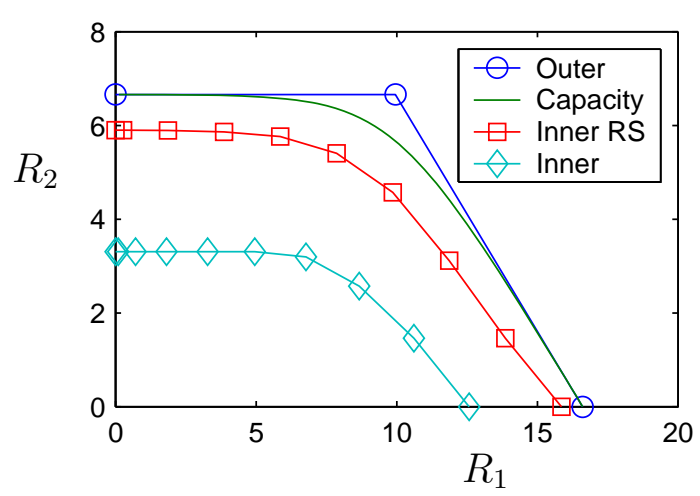

(b)

Figure 5: AWGN broadcast channel rate regions: (a) users 1 and 2 have SNRs $20 \mathrm{~dB}$ and $10 \mathrm{~dB}$. (b) users 1 and 2 have SNRs $50 \mathrm{~dB}$ and $20 \mathrm{~dB}$. Each plot compares the outer bound, the capacity, and the inner bounds with reverse stripping (RS) and without.

Note that (101) identifies the extreme points $\left(C_{1}, 0\right)$ and $\left(C_{1}-\rho C_{2}, C_{2}\right)$. Note that the complete outer bound region also includes the corner point $\left(C_{1}, 0\right)$ that is otherwise dominated in the weighted sum rate $R_{1}+\omega R_{2}$ by the extreme point $\left(C_{1}-\rho C_{2}, C_{2}\right)$.

For the achievable rates of BES signaling, we observe from (80) that signals levels 1 through $n_{2}^{*} \approx(1 / 2) \log s_{2}^{*}$ are useful to both receivers while levels above $n_{2}^{*}$ can transmit data only to receiver 1 . However, we note that using those high levels for user 1 does subject user 2 to the penalty of additional LSB interference. In particular, to maximize the gain from reverse stripping, we assign levels up to $n_{2}$ to user 2 and levels above $n_{2}$ to user 1 . Thus user 1 faces zero LSB interference. By varying $n_{2}$, we obtain from Theorem 5 the boundary points $\left(R_{1}, R_{2}\right)$ of an achievable rate region given by

$$
\begin{aligned}
& R_{1}=2 p_{1} \sum_{n=n_{2}+1}^{\infty} \hat{H}_{\infty}\left(3 s_{1}^{*} 2^{-2 n}\right) \\
& R_{2}=2 p_{2} \sum_{n=1}^{n_{2}} \hat{H}_{n_{2}-n}\left(3 s_{2}^{*} 2^{-2 n}\right)
\end{aligned}
$$

Note that if reverse stripping is not implemented at the receivers, the achievable rates are reduced to

$$
\begin{aligned}
& R_{1}=2 p_{1} \sum_{n=n_{2}+1}^{\infty} \hat{H}_{0}\left(3 s_{1}^{*} 2^{-2 n}\right) \\
& R_{2}=2 p_{2} \sum_{n=1}^{n_{2}} \hat{H}_{0}\left(3 s_{2}^{*} 2^{-2 n}\right)
\end{aligned}
$$




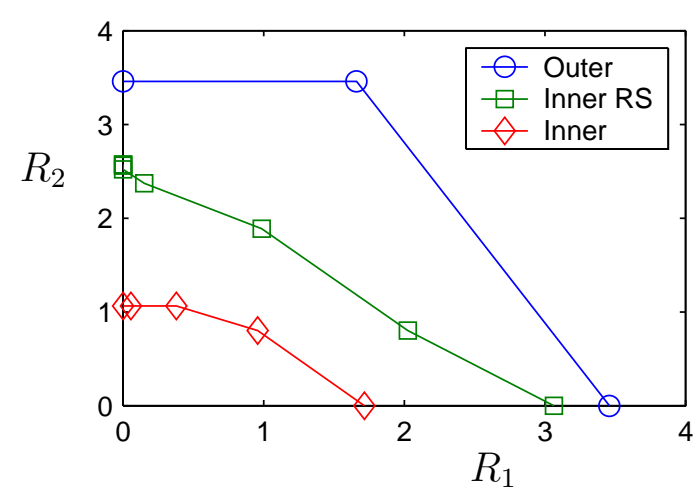

(a)

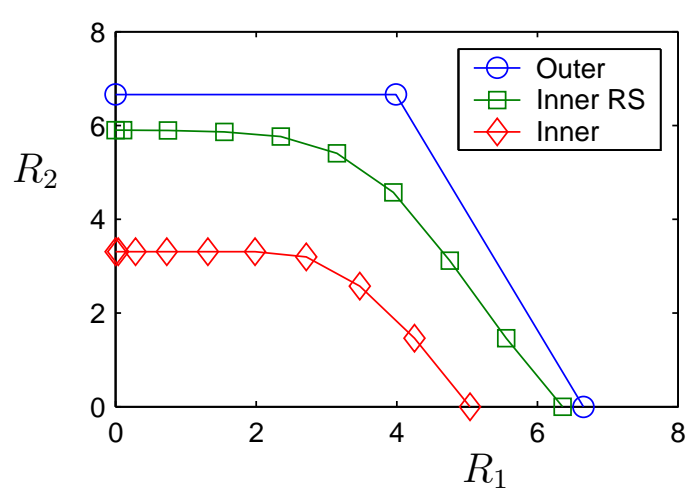

(b)

Figure 6: AWGN broadcast channel rate regions: (a) users 1 and 2 have SNRs $20 \mathrm{~dB}$ and $10 \mathrm{~dB}$. (b) users 1 and 2 have SNRs $50 \mathrm{~dB}$ and $20 \mathrm{~dB}$. In each case, the user 2 channel is good with probability $p_{2}$. Each plot compares the outer bound, the capacity, and the inner bounds with reverse stripping (RS) and without.

For numerical comparisons, we start with the ordinary AWGN BC in which $p_{1}=$ $p_{2}=1$ in order to assess the inner and outer bounds when the capacity region is known [4]. Figure 5 considers high SNR examples in which (a) user 1 has SNR $10 \log _{10} s_{1}^{*}=20 \mathrm{~dB}$ while user 2 has SNR $10 \mathrm{~dB}$ and (b) user 1 has SNR $50 \mathrm{~dB}$ and user 2 has SNR $20 \mathrm{~dB}$. Each plot compares the outer bound (101), the capacity given by Gaussian superposition codes, the inner bound (102) with reverse stripping and the inner bound (103) without reverse stripping. We see that the outer bound is within one bit of the capacity boundary, an observation appearing first in [11]. With reverse stripping, the BES achievable rates are also within one bit of the capacity region. However, in the absence of reverse stripping, the BES scheme is penalized considerably, although this penalty is dimishing with increasing SNR.

Next we consider a corresponding pair of broadcast channels in which user 2 has the same AWGN channel with $p_{2}=1$ but user 1 now has a channel activity factor $p_{1}<1$ such that users 1 and 2 have equal ergodic capacities $C_{1}=C_{2}$. In this case, numerical comparisons of the inner and outer bounds are given in Figure 6. In these cases, user 1 has activity factor (a) $p_{1} \approx 0.52$ and (b) $p_{1}=0.4$, In comparing Figures 5 and 6 , we see little qualititative difference. Essentially, both the inner and outer bounds reflect that user 2 has access to a high SNR channel with probability $p_{1}$. This activity factor simply scales the bits rates achieved by user 2 . The fundamental policy that the LSBs are reserved for user 2 remains the same.

\section{Intermittent AWGN channel vs. Rayleigh fading channel}

Now consider the case when user 1 still has an intermittent AWGN channel described by the activity probability $p_{1}$ and good channel SNR $s_{1}^{*}$ but user 2 has a Rayleigh 
fading channel state $S_{2}$ with average SNR $\Gamma_{2}$ and thus complementary CDF

$$
\bar{F}_{S_{2}}(s)= \begin{cases}1 & s<0 \\ e^{-s / \Gamma_{2}} & s \geq 0\end{cases}
$$

For the outer bound, the partition (57) yields

$$
\begin{aligned}
& \mathcal{I}_{1}(\omega)= \begin{cases}{\left[0, s_{1}^{*}\right]} & 0 \leq \omega<p_{1}, \\
\left(\Gamma_{2} \ln \left(\omega / p_{1}\right), s_{1}^{*}\right] & p_{1} \leq \omega<p_{1} e^{s_{1}^{*} / \Gamma_{2}} \\
\phi & e^{s_{1}^{*} / \Gamma_{2}} \leq \omega,\end{cases} \\
& \mathcal{I}_{2}(\omega)= \begin{cases}\left(s_{1}^{*}, \infty\right) & \omega<p_{1} \\
{\left[0, \Gamma_{2} \ln \left(\omega / p_{1}\right)\right] \cup\left(s_{1}^{*}, \infty\right)} & p_{1} \leq \omega<p_{1} e^{s_{1}^{*} / \Gamma_{2}}, \\
{[0, \infty)} & p_{1} e^{s_{1}^{*} / \Gamma_{2}} \leq \omega .\end{cases}
\end{aligned}
$$

We see in 105 that varying the weight $\omega$ over $\left[p_{1}, p_{1} e^{s_{1}^{*} / \Gamma_{2}}\right]$ simply corresponds to varying a threshold channel state

$$
s_{\omega}:=\Gamma_{2} \ln \frac{\omega}{p_{1}}
$$

over the interval $\left[0, s_{1}^{*}\right]$. This permits us to write the channel state partition as

$$
\begin{aligned}
& \mathcal{I}_{1}\left(s_{\omega}\right)=\left(s_{\omega}, s_{1}^{*}\right] \\
& \mathcal{I}_{2}\left(s_{\omega}\right)=\left[0, s_{\omega}\right] \cup\left(s_{1}^{*}, \infty\right)
\end{aligned}
$$

To describe the outer bound region of Theorem 3, we define the integral function

$$
\mu_{\Gamma}(a, b):=\log e \int_{a}^{b} e^{-s / \Gamma} \frac{1}{1+s} d s .
$$

The outer bound region is then specified by a continuous boundary of extreme points $\left(R_{1}^{*}\left(s_{\omega}\right), R_{2}^{*}\left(s_{\omega}\right)\right)$ found by varying $s_{\omega}$ over the interval $\left[0, s_{1}^{*}\right]$. From 105 , these boundary points are given by

$$
\begin{aligned}
& R_{1}^{*}\left(s_{\omega}\right)=p_{1} \log \left[\frac{1+s_{1}^{*}}{1+s_{\omega}}\right], \\
& R_{2}^{*}\left(s_{\omega}\right)=\mu_{\Gamma_{2}}\left(0, s_{\omega}\right)+\mu_{\Gamma_{2}}\left(s_{1}^{*}, \infty\right) .
\end{aligned}
$$

This outer bound is shown in Figure 7 for an instance in which receiver 1 has an intermittent AWGN channel $S_{1}$ with activity probability $p_{1}=0.4$ and maximum SNR $s_{1}^{*}$ of $60 \mathrm{~dB}$.

For the BES achievability rates, we observe that user 1 has maximum SNR $s_{1}^{*}$ and thus only signal levels $n \leq n_{1}^{*}:=\hat{n}\left(s_{1}^{*}\right)$ can be used to communicate to receiver 1. Hence signal levels $n>n_{1}^{*}$ are assigned to user 2. However, signal levels $n \leq n_{1}^{*}$ may be useful for communication to either receiver. In particular, we observe from the outer bound that when the weight $\omega$ is sufficiently large, signal levels $s$ below a threshold were associated with receiver 2 . Hence for our achievability scheme, we 


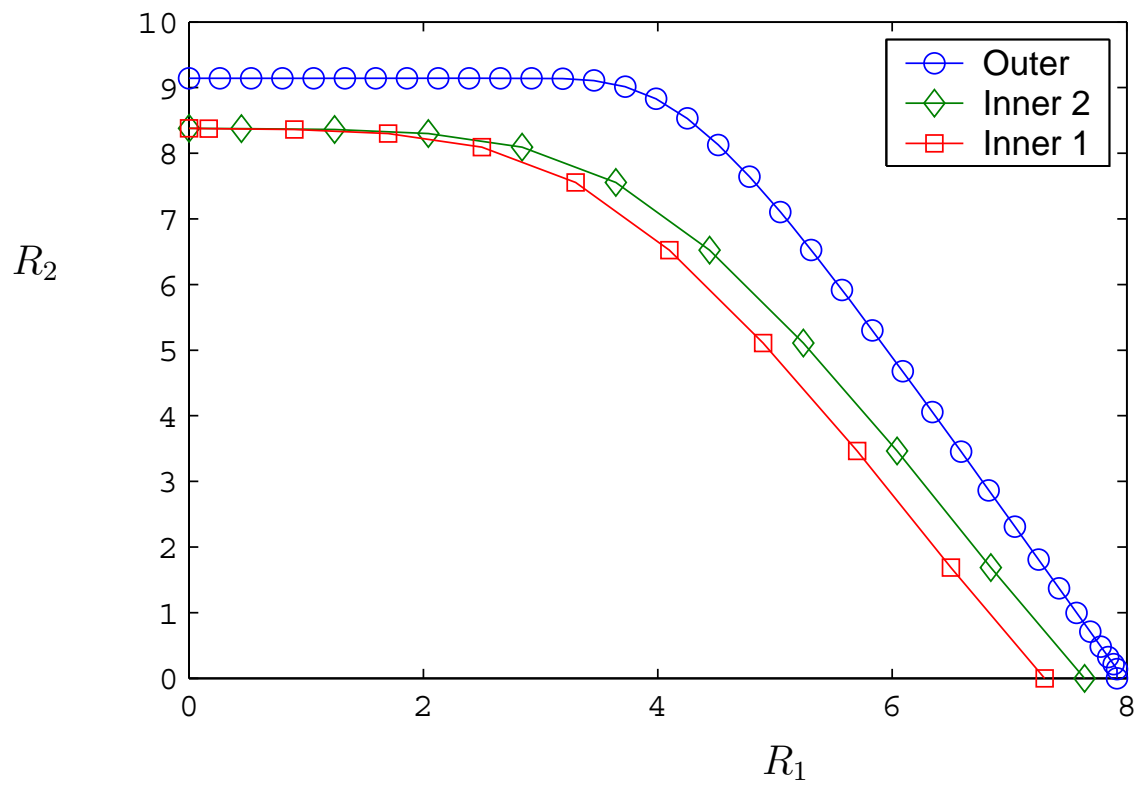

Figure 7: Inner and outer bounds when user 1 has an intermittent AWGN channel with activity factor 0.4 and maximum SNR $60 \mathrm{~dB}$ and user 2 has a Rayleigh fading channel with $30 \mathrm{~dB}$ average SNR.

assign signal levels $n \in\left\{1, \ldots, n_{2}\right\}$ to user 2 and signal levels $n \in\left\{n_{2}+1, \ldots, n_{1}^{*}\right\}$ to user 1. That is, for a given threshold $n_{1}$, we obtain achievable rates from Theorem 5 with

$$
\begin{aligned}
& \hat{\mathcal{N}}_{1}=\left\{n_{2}+1, \ldots, n_{1}^{*}\right\}, \\
& \hat{\mathcal{N}}_{2}=\left\{1, \ldots, n_{2}\right\} \cup\left\{n_{1}^{*}+1, n_{1}^{*}+2, \ldots\right\} .
\end{aligned}
$$

We note that user 2 suffers zero LSB interference for levels $n>n_{1}^{*}$. Furthermore each receiver can also employ reverse stripping. Varying the threshold $n_{2}$, Theorem 5 yields the achievable rate pairs

$$
\begin{aligned}
& R_{1}=2 p_{1} \sum_{n=n_{2}+1}^{n_{1}^{*}} \hat{H}_{n_{1}^{*}-n}\left(3 s_{1}^{*} 2^{-2 n}\right) \\
& R_{2}=2 \sum_{n=1}^{n_{2}} \mathrm{E}_{S_{2}}\left[\hat{H}_{n_{2}-n}\left(3 S_{2} 2^{-2 n}\right)\right]+2 \sum_{n=n_{1}^{*}+1}^{\infty} \mathrm{E}_{S_{2}}\left[\hat{H}_{\infty}\left(3 S_{2} 2^{-2 n}\right)\right] .
\end{aligned}
$$

In Figure 7, these rates are tagged "Inner 1" and they are seen to be somewhat worse than an alternate "Inner 2" achievable scheme. Note that both schemes employ reverse stripping. For the "Inner 2" scheme, we make the observation that signals levels $n>n_{1}^{*}$ which, following the guidance of the outer bound assignment, are assigned to receiver 2 actually convey negligible information. In fact, information is 
conveyed to user 2 on these signal levels only when the user 1 SNR exceeds $60 d B$, which is rare since user 2 has an average SNR of $30 \mathrm{~dB}$. However assigning these levels to user 2 penalizes the reverse stripping mechanism of receiver 1 . In particular, by not transmitting on these levels, user 1 will face no LSB interference and thus will be able to obtain the "Inner 2" rates

$$
\begin{aligned}
& R_{1}=2 p_{1} \sum_{n=n_{2}+1}^{n_{1}^{*}} \hat{H}_{\infty}\left(3 s_{1}^{*} 2^{-2 n}\right), \\
& R_{2}=2 \sum_{n=1}^{n_{2}} \mathrm{E}_{S_{2}}\left[\hat{H}_{n_{2}-n}\left(3 S_{2} 2^{-2 n}\right)\right] .
\end{aligned}
$$

We see in Figure 7 that this provides receiver 1 a half-bit rate increase when the transmitter is largely dedicated to receiver 1 with a neglible reduction to receiver 2 .

Note however that the edge for "Inner 2" strategy ceases if receiver 2 has sufficiently high average SNR to exploit the high levels. More generally, we observe that following the guidance of the outer bound may not maximize the BES rates. In fact, one could directly optimize the BES achievable rates. However, even the simple example of Figure 7 shows there are non-obvious tradeoffs between the allocation of signal levels and the rate improvements afforded by enhancements to reverse stripping.

\section{Fading Gaussian BC: A Constant Gap Result}

With knowledge of the distributions $\bar{F}_{S_{i}}(s)$, Theorems 3 and 5 can be used for direct calculation of outer and inner bounds to the fading $\mathrm{BC}$ capacity region. Now we show that the achievable rates of Theorem 4 are within a constant gap of the outer bound rates of Theorem 3 for all channel state distributions.

The key idea in matching up the inner and outer bounds is a quantization of the channel state analagous to the signal levels of layered erasure channel. In particular, we let $\gamma>0$ denote a constant to be determined later and define the channel states

$$
\begin{aligned}
\gamma_{n} & =\gamma 2^{2(n-1)}, & n & =1,2, \ldots, \\
\Gamma_{n} & =\left[\gamma_{n}, \gamma_{n+1}\right), & n & =1,2, \ldots
\end{aligned}
$$

In the absence of specific distributions for $S_{1}$ and $S_{2}$, we enlarge the Theorem 3 outer bound by enhancing and discretizing the channel gains $S_{1}$ and $S_{2}$. We define the enhanced channels $\bar{S}_{1}$ and $\bar{S}_{2}$ by the complementary CDFs

$$
\bar{F}_{\bar{S}_{i}}(s)= \begin{cases}1 & s \leq \gamma \\ \bar{F}_{S_{i}}\left(\gamma_{n}\right) & s \in \Gamma_{n}, \quad n=1,2, \ldots\end{cases}
$$

Since $\bar{F}_{\bar{S}_{i}}(s) \geq \bar{F}_{S_{i}}(s)$, the outer bound of Theorem 3 correpsonding to the fading Gaussian BC $\left(\bar{S}_{1}, \bar{S}_{2}\right)$ contains the Theorem 3 outer bound region of the fading Gaussian $\mathrm{BC}\left(S_{1}, S_{2}\right)$. For this enhanced channel, the extreme points of the rate region 
are given by

$$
\begin{aligned}
& \bar{R}_{1}=\log e \int_{\mathcal{I}_{1}(\omega)} \bar{F}_{\bar{S}_{1}}(s) \frac{1}{1+s} d s \\
& \bar{R}_{2}=\log e \int_{\mathcal{I}_{2}(\omega)} \bar{F}_{\bar{S}_{2}}(s) \frac{1}{1+s} d s .
\end{aligned}
$$

with $\mathcal{I}_{i}(\omega)$ given by 57 with $S_{i}$ replaced by the enhanced channel $\bar{S}_{i}$. However, because $\bar{F}_{\bar{S}_{i}}(s)$ is constant over each interval $\Gamma_{n}$,

$$
\mathcal{I}_{i}(\omega) \subseteq[0, \gamma) \cup \bigcup_{n \in \mathcal{N}_{i}(\omega)} \Gamma_{n}
$$

such that

$$
\begin{aligned}
& \mathcal{N}_{1}(\omega)=\left\{n \geq 1 \mid \bar{F}_{S_{1}}\left(\gamma_{n}\right)>\omega \bar{F}_{S_{2}}\left(\gamma_{n}\right)\right\} \\
& \mathcal{N}_{2}(\omega)=\left\{n \geq 1 \mid \bar{F}_{S_{1}}\left(\gamma_{n}\right) \leq \omega \bar{F}_{S_{2}}\left(\gamma_{n}\right)\right\}
\end{aligned}
$$

Since $\bar{F}_{\bar{S}_{i}}(s)=1$ for $s \leq \gamma$, and is constant over each interval $\Gamma_{n}$, it follows from (116) that

$$
\bar{R}_{i} \leq \log (1+\gamma)+\sum_{n \in \mathcal{N}_{i}(\omega)} \bar{F}_{S_{i}}\left(\gamma_{n}\right)[C(n+1)-C(n)]
$$

where

$$
C(n)=\log \left(1+\gamma_{n}\right)
$$

is the capacity of the point-to-point channel with $\operatorname{SNR} \gamma_{n}$. We note that

$$
\Delta_{C}(n):=C(n+1)-C(n)
$$

is the incremental capacity associated with the channel improving from state $\gamma_{n}$ to state $\gamma_{n+1}$. Note that $\Delta_{C}(n) \leq 2$ and approaches this upper bound when $n \rightarrow \infty$. This roughly matches the binary expansion model of Section 3 , in that going from state $n$ to state $n+1$ in the complex channel yields approximately one additional bit each for the in-phase and quadrature channels. Applying the bound $\Delta_{C}(n) \leq 2$ to (119), we obtain the outer bound

$$
R_{i} \leq \log (1+\gamma)+2 \sum_{n \in \mathcal{N}_{i}(\omega)} \bar{F}_{S_{i}}\left(\gamma_{n}\right)
$$

For the purpose of a constant gap result, we start with BES achievable rate without reverse stripping. From (81), user $i$ can communicate reliably on level $n \in \hat{\mathcal{N}}_{i}$ at rate

$$
\begin{aligned}
r_{n}^{(i)} & \geq 2 \int_{\gamma_{n}}^{\infty} f_{S_{i}}(s)\left[1-H\left(\hat{\epsilon}_{0}\left(a_{n}(s)\right)\right)\right] d s \\
& =2 \bar{F}_{S_{i}}\left(\gamma_{n}\right)-2 \delta_{n}^{(i)} .
\end{aligned}
$$


where

$$
\delta_{n}^{(i)}=\sum_{k=n}^{\infty} \int_{\Gamma_{k}} f_{S_{i}}(s) H\left(\hat{\epsilon}_{0}\left(a_{n}(s)\right)\right) d s .
$$

Note that $a_{n}(s)$ is an increasing function of $s$ and $\hat{\epsilon}_{0}(a)$ is a decreasing function of $a$. Thus $\hat{\epsilon}_{0}\left(a_{n}(s)\right)$ is a decreasing function of $s$. Since $\hat{\epsilon}_{0}\left(a_{n}(s)\right) \leq 1 / 2, H\left(\hat{\epsilon}_{0}\left(a_{n}(s)\right)\right)$ is a decreasing function of $s$. Thus for $s \in \Gamma_{k}$,

$$
H\left(\hat{\epsilon}_{0}\left(a_{n}(s)\right)\right) \leq H\left(\hat{\epsilon}_{0}\left(a_{n}\left(\gamma_{k}\right)\right)\right)=H\left(\hat{\epsilon}_{0}\left(3 \gamma_{k-n}\right)\right) .
$$

This implies

$$
\begin{aligned}
\delta_{n}^{(i)} & \leq \sum_{k=n}^{\infty} H\left(\hat{\epsilon}_{0}\left(3 \gamma_{k-n}\right)\right) P\left[S_{i} \in \Gamma_{k}\right] \\
& \leq \sum_{m=0}^{\infty} H\left(\hat{\epsilon}_{0}\left(3 \gamma_{m}\right)\right) P\left[S_{i} \in \Gamma_{m+n}\right] .
\end{aligned}
$$

With the assignment of signal levels $n \in \hat{\mathcal{N}}_{i}$, user $i$ can achieve a rate

$$
R_{i}=2 \sum_{n \in \hat{\mathcal{N}}_{i}} r_{n}^{(i)} \geq 2 \sum_{n \in \hat{\mathcal{N}}_{i}} \bar{F}_{S_{i}}\left(\gamma_{n}\right)-\delta^{(i)},
$$

where

$$
\delta^{(i)}=\sum_{n \in \hat{\mathcal{N}}_{i}} \delta_{n}^{(i)}
$$

satisfies the upper bound

$$
\begin{aligned}
\delta^{(i)} \leq \sum_{n=1}^{\infty} \delta_{n}^{(i)} & =\sum_{n=1}^{\infty} \sum_{m=0}^{\infty} H\left(\hat{\epsilon}_{0}\left(3 \gamma_{m}\right)\right) P\left[S_{i} \in \Gamma_{m+n}\right] \\
& =\sum_{m=0}^{\infty} H\left(\hat{\epsilon}_{0}\left(3 \gamma_{m}\right)\right) \bar{F}_{S_{i}}\left(\gamma_{m+1}\right) \\
& \leq \sum_{m=0}^{\infty} H\left(\hat{\epsilon}_{0}\left(3 \gamma_{m}\right)\right) .
\end{aligned}
$$

In terms of the channel state set $\left\{\gamma_{n}\right\}$, we obtain the inner bound

$$
R_{i} \geq 2 \sum_{n \in \hat{\mathcal{N}}_{i}} \bar{F}_{S_{i}}\left(\gamma_{n}\right)-2 \sum_{m=0}^{\infty} H\left(\hat{\epsilon}_{0}\left(3 \gamma_{m}\right)\right) .
$$

In comparing the inner bound (134) to the outer bound (122), the rate gap for user $i$ is given by

$$
\Delta_{i}=\log (1+\gamma)+2 \sum_{m=0}^{\infty} H\left(\hat{\epsilon}_{0}\left(3 \gamma_{m}\right)\right)
$$


As $\gamma_{m}=\gamma 2^{2(m-1))}$, this bound can be minimized by choosing $\gamma=5.65$, yielding $\Delta_{i} \leq 6.386$. This is a universal gap that holds for all channel state distributions. This is significantly larger than the gaps observed in the examples of Section 4.4 because this is a worst-case bound on the gap over all fading distributions. We note that our specific examples showed the reverse stripping scheme can deliver a 2-3 bit improvement in bit rates. In the worst case, however, reverse stripping is unlikely to help because the transmission scheme may end up assigning every other level to a user.

On the other hand, there is still some potential on improving even the worstcase gap. We observe that in the quantization of channel states in $6 \mathrm{~dB}$ steps, the outer bound is loosened by assuming a receiver always obtains the best channel in each interval while the inner bound is tightened by the opposite assumption that a receiver always gets the worst channel. The impact of quantization appears to be on the order of two bits. Thus we conjecture that the actual worst-case gap is considerably smaller than six bits. Nevertheless, the 6.386 bit gap does demonstrate that the BES signaling has the right asymptotic behavior in high SNR.

\section{Conclusion}

This work derives the first constant gap result for the capacity region of the AWGN fading broadcast channel with channel state information known at the receivers only. Our calculations show that the rate gap can then be bounded by $6.386 \mathrm{bits} / \mathrm{s} / \mathrm{Hz}$ universally for all fading distributions. To obtain this conclusion, we derive a new outer bound and use a simple achievability strategy, both of which are motivated by the analysis of an approximating layered erasure broadcast channel. We conjecture that more careful analysis and more sophisticated achievability schemes will shrink this gap considerably.

\section{A Proofs}

\section{Proof: Lemma 1}

(a) As $N$ is a deterministic function of $X^{N}$,

$$
\begin{aligned}
I\left(X^{q} ; X^{N} \mid V\right) & =I\left(X^{q} ; X^{N}, N \mid V\right) \\
& =I\left(X^{q} ; N \mid V\right)+I\left(X^{q} ; X^{N} \mid V, N\right) .
\end{aligned}
$$

Since the channel state $N$ is independent of $V$ and $X^{q}, I\left(X^{q} ; N \mid V\right)=0$. Thus

$$
\begin{aligned}
I\left(X^{q} ; X^{N} \mid V\right) & =I\left(X^{q} ; X^{N} \mid V, N\right) \\
& =H\left(X^{N} \mid V, N\right),
\end{aligned}
$$

since $H\left(X^{N} \mid V, N, X^{q}\right)=0$. 
(b) Since $V$ and $X^{n}$ are independent of $N$,

$$
\begin{aligned}
H\left(X^{N} \mid V, N\right) & =\sum_{n=1}^{q} P_{N}(n) H\left(X^{n} \mid V, N=n\right) \\
& =\sum_{n=1}^{q} P_{N}(n) H\left(X^{n} \mid V\right) .
\end{aligned}
$$

Applying the chain rule and reversing the order of summation then yields

$$
\begin{aligned}
H\left(X^{N} \mid V, N\right) & =\sum_{n=1}^{q} \sum_{j=1}^{n} P_{N}(n) H\left(X_{j} \mid X^{j-1}, V\right) \\
& =\sum_{j=1}^{q} \sum_{n=j}^{q} P_{N}(n) H\left(X_{j} \mid X^{j-1}, V\right) \\
& =\sum_{j=1}^{q} \bar{F}_{N}(j) H\left(X_{j} \mid X^{j-1}, V\right) .
\end{aligned}
$$

(c) As $N$ is a deterministic function of $X^{N}$,

$$
\begin{aligned}
I\left(V ; X^{N}\right) & =I\left(V ; X^{N}, N\right) \\
& =I(V ; N)+I\left(V ; X^{N} \mid N\right) .
\end{aligned}
$$

Since $V$ is independent of the channel state $N, I(V ; N)=0$ and

$$
\begin{aligned}
I\left(V ; X^{N}\right) & =I\left(V ; X^{N} \mid N\right) \\
& =H\left(X^{N} \mid N\right)-H\left(X^{N} \mid V, N\right) .
\end{aligned}
$$

Applying the result of part (a) to $H\left(X^{N} \mid N\right)$ (with a trivial $V$ ) and also to $H\left(X^{N} \mid V, N\right)$ yields

$$
\begin{aligned}
I\left(V ; X^{N}\right) & =\sum_{j=1}^{q} \bar{F}_{N}(j)\left[H\left(X_{j} \mid X^{j-1}\right)-H\left(X_{j} \mid V, X^{j-1}\right)\right] \\
& =\sum_{j=1}^{q} \bar{F}_{N}(j) I\left(V ; X_{j} \mid X^{j-1}\right) .
\end{aligned}
$$

Proof: Lemma 4 From 43), independence of $X$ and $\tilde{S}_{1}$ and independence of $V$ and $S_{2}$ imply

$$
\begin{aligned}
R^{*}(\omega) & \leq \max _{V, X} I\left(X ; Y_{1} \mid V, \tilde{S}_{1}\right)+\omega I\left(V ; Y_{2} \mid S_{2}\right) \\
& =\max _{V, X} h\left(Y_{1} \mid V, \tilde{S}_{1}\right)-h\left(Z_{1}\right)+\omega\left[h\left(Y_{2} \mid S_{2}\right)-h\left(Y_{2} \mid V, S_{2}\right)\right] \\
& =\max _{V, X} h\left(Y_{1} \mid V, \tilde{S}_{1}\right)-\omega h\left(Y_{2} \mid V_{1}, S_{2}\right)+\omega h\left(Y_{2} \mid S_{2}\right)-h(Z),
\end{aligned}
$$


In terms of $Y^{(s)}$, we can write

$$
\begin{aligned}
h\left(Y_{1} \mid V, \tilde{S}_{1}\right) & =\int_{0}^{\infty} f_{\tilde{S}_{1}}(s) h(\sqrt{s} X+Z \mid V, \tilde{S}=s) d s \\
& =\int_{0}^{\infty} f_{\tilde{S}_{1}}(s) h(\sqrt{s} X+Z \mid V) d s \\
& =\int_{0}^{\infty} f_{\tilde{S}_{1}}(s) h\left(Y^{(s)} \mid V\right) d s
\end{aligned}
$$

Similarly,

$$
h\left(Y_{2} \mid V, S_{2}\right)=\int_{0}^{\infty} f_{S_{2}}(s) h\left(Y^{(s)} \mid V\right) d s
$$

and

$$
\begin{aligned}
h\left(Y_{2} \mid S_{2}\right) & =\int_{0}^{\infty} f_{S_{2}}(s) h\left(\sqrt{s} X+Z_{2} \mid S_{2}=s\right) d s \\
& \leq \int_{0}^{\infty} f_{S_{2}}(s) \frac{1}{2} \log [2 \pi e(s+1)] d s \\
& =h(Z)+C_{e}\left(S_{2}\right) .
\end{aligned}
$$

Applying 156, 157) and 160 to 153, we obtain

$$
R^{*}(\omega) \leq \max _{V, X} \int_{0}^{\infty} f_{\omega}(s) h\left(Y^{(s)} \mid V\right) d s-(1-\omega) h(Z)+\omega C_{e}\left(S_{2}\right)
$$

where $f_{\omega}(s)=f_{\tilde{S}_{1}}(s)-\omega f_{S_{2}}(s)$. Since $f_{\omega}(s)=-d \bar{F}_{\omega}(s) / d s$, integration by parts can be used to show that for any $g(s)$ satisfying $\bar{F}_{\omega}(\infty) g(\infty)=0$ that

$$
\int_{0}^{\infty} f_{\omega}(s) g(s) d s=\bar{F}_{\omega}(0) g(0)+\int_{0}^{\infty} \bar{F}_{\omega}(s) g^{\prime}(s) d s .
$$

Note that $\bar{F}_{\omega}(0)=1-\omega$ and that $g(s)=h\left(Y^{(s)} \mid V\right)$ implies $g(0)=\left.h\left(Y^{(s)} \mid V\right)\right|_{s=0}=$ $h(Z)$. This implies

$$
\int_{0}^{\infty} f_{\omega}(s) h\left(Y^{(s)} \mid V\right)=(1-\omega) h(Z)+\int_{0}^{\infty} \bar{F}_{\omega}(s) h^{\prime}\left(Y^{(s)} \mid V\right) d s,
$$

where $h^{\prime}\left(Y^{(s)} \mid v\right)$ denotes the derivative of $h\left(Y^{(s)} \mid V\right)$ with respect to $s$. It follows from (161) and 163) that

$$
R^{*}(\omega) \leq \max _{V, X} \int_{0}^{\infty} \bar{F}_{\omega}(s) h^{\prime}\left(Y^{(s)} \mid V\right) d s+\omega C_{e}\left(S_{2}\right)
$$

The claim follows since $I\left(X ; Y^{(s)} \mid V\right)=h\left(Y^{(s)} \mid V\right)-h(Z)$, implies $I^{\prime}\left(X ; Y^{(s)} \mid V\right)=$ $h^{\prime}\left(Y^{(s)} \mid V\right)$. 
Proof: Lemma 6 From 94,

$$
\begin{aligned}
P\left[C\left(\tilde{x}^{n}\right)\right] & =P\left[2^{-n}\left(-1-2^{n-d} \tilde{U}_{n}\right) \leq \tilde{Z}<2^{-n}\left(1-2^{n-d} \tilde{U}_{n}\right)\right] \\
& =P\left[-1+U \leq Z_{n}<1+U\right]
\end{aligned}
$$

where $U=-2^{n-d} U_{n}$ is a continuous uniform $\left(-2^{-d}, 2^{-d}\right)$ random variable and $Z_{n}=$ $2^{n} \tilde{Z}$ has standard deviation $\sigma_{n}=2^{n} / \sqrt{3 s}=1 / \sqrt{a_{n}(s)}$. Since $U$ and $Z_{n}$ are independent,

$$
\begin{aligned}
P\left[C\left(\tilde{x}^{n}\right)\right] & =2^{d-1} \int_{-2^{-d}}^{2^{-d}} P\left[-1+u \leq Z_{n}<1+u\right] d u \\
& =2^{d-1} \int_{-2^{-d}}^{2^{-d}}\left[1-Q\left(\frac{1+u}{\sigma_{n}}\right)-Q\left(\frac{1-u}{\sigma_{n}}\right)\right] d u \\
& =1-\sigma_{n} 2^{d} \int_{\left(1-2^{-d}\right) / \sigma_{n}}^{\left(1+2^{-d}\right) / \sigma_{n}} Q(v) d v .
\end{aligned}
$$

Noting that $G(x)=\int Q(x) d x$ and that $\sigma_{n}=1 / \sqrt{a_{n}(s)}$, we obtain

$$
1-P\left[C\left(\tilde{x}^{n}\right)\right]=\frac{G\left(\sqrt{a_{n}(s)}\left(1+2^{-d}\right)\right)-G\left(\sqrt{a_{n}(s)}\left(1-2^{-d}\right)\right)}{\sqrt{a_{n}(s)} 2^{-d}}=\epsilon_{d}\left(a_{n}(s)\right) .
$$

Since the conditional probability $P\left[C\left(\tilde{x}^{n}\right)\right]$ does not depend on $\tilde{x}^{n}$, it follows that the error (i.e. crossover) probability of this detector for bit $\tilde{X}_{n}$ under channel state $S=s$ with LSB interference at depth $d$ satisfies

$$
p_{n, d}(s) \leq P\left[\hat{X}_{n} \neq \tilde{X}_{n}\right] \leq 1-P\left[C\left(\tilde{x}^{n}\right)\right]=\epsilon_{d}\left(a_{n}(s)\right) .
$$

The claim follows by using 75 to guess bits when the channel state is weak.

\section{References}

[1] R. Ahlswede. Multi-way communication channels. In Proc. 2nd Int. Symp. Inform. Theory (1971), pages 23-52, Tsahkadsor, Armenian S.S.R., 1973. Publishing House of the Hungarian Academy of Sciences.

[2] H. Liao. Multiple access channels. PhD thesis, University of Hawaii, Honolulu, Dept. of Electrical Engineering, 1972.

[3] T. M. Cover. Broadcast channels. IEEE Trans. Info. Theory, 18(1):2-14, January 1972.

[4] P. P. Bergmans. A simple converse for broadcast channels with additive white Gaussian noise. IEEE Trans. Info. Theory, 20:279-280, Mar 1974. 
[5] D. Hughes-Hartogs. The capacity of a degraded spectral Gaussian broadcast channel. PhD thesis, Inform. Syst. Lab., Ctr. Syst. Res., Stanford University, Stanford, CA, July 1975.

[6] D. N. C. Tse. Optimal power allocation over parallel Gaussian broadcast channels. In IEEE Intl. Symp. Info. Theory ISIT, page 27, Ulm, Germany, June 1997.

[7] L. Li and A. Goldsmith. Capacity and optimal resource allocation for fading broadcast channels .i. ergodic capacity. IEEE Trans. Info. Theory, 47(3):10831102, March 2001.

[8] H. Weingarten, Y. Steinberg, and S. Shamai (Shitz). The capacity region of the Gaussian multiple-input multiple-output broadcast channel. IEEE Trans. Info. Theory, 52(9):3936-3964, 2006.

[9] D. Tuninetti and S. Shamai. On two-user fading Gaussian broadcast channels with perfect channel state information at the receivers. In IEEE Intl. Symp. Info. Theory ISIT, Yokohama, Japan, July 2003.

[10] A. Jafarian and S. Vishwanath. On the capacity of one-sided two user gaussian fading broadcast channels. In Proc. Globecom, Dec 2008.

[11] A. S. Avestimehr, S. Diggavi, and D. Tse. A deterministic approach to wireless relay networks. In Proceedings of Allerton Conference, 2007.

[12] S. Bhadra, P. Gupta, and S. Shakkottai. On network coding for interference networks. In IEEE Intl. Symp. Info. Theory ISIT, pages 207-211, 2006.

[13] N. Martins, M. Dahleh, and N. Elia. Feedback stabilization of uncertain systems in the presence of a direct link. IEEE Transactions on Automatic Control, 51(3):438-447, March 2006.

[14] R. G. Gallager. Capacity and coding for degraded broadcast channels. Probl. Pered. Inform., 10(3):3-14, July-Sept. 1974. translated in Probl. Inform. Transm., pp. 185-193, July-Sept. 1974.

[15] K. Marton. A coding theorem for the discrete memoryless broadcast channel. IEEE Trans. Info. Theory, 25(1):306-311, may 1979.

[16] D. Guo, S. Shamai, and S. Verdu. Mutual information and minimum meansquare error in Gaussian channels. IEEE Trans. Info. Theory, 51:1261-1282, April 2005. 